\title{
Hydraulic adjustment to an obstacle in a rotating channel
}

\author{
By L. J. PRATT ${ }^{1}$ K. R. HELFRICH ${ }^{1}$ \\ AND E. P. CHASSIGNET ${ }^{2}$ \\ ${ }^{1}$ Department of Physical Oceanography, Woods Hole Oceanographic Institution, Woods Hole, \\ MA 02543, USA \\ ${ }^{2}$ Department of Meteorology and Physical Oceanography, RSMAS, University of Miami, \\ Miami, FL 33146, USA
}

(Received 17 March 1999 and in revised form 8 September 1999)

In order to gain insight into the hydraulics of rotating-channel flow, a set of initialvalue problems analogous to Long's towing experiments is considered. Specifically, we calculate the adjustment caused by the introduction of a stationary obstacle into a steady, single-layer flow in a rotating channel of infinite length. Using the semigeostrophic approximation and the assumption of uniform potential vorticity, we predict the critical obstacle height above which upstream influence occurs. This height is a function of the initial Froude number, the ratio of the channel width to an appropriately defined Rossby radius of deformation, and a third parameter governing how the initial volume flux in sidewall boundary layers is partitioned. (In all cases, the latter is held to a fixed value specifying zero flow in the right-hand (facing downstream) boundary layer.) The temporal development of the flow according to the full, two-dimensional shallow water equations is calculated numerically, revealing numerous interesting features such as upstream-propagating shocks and separated rarefying intrusions, downstream hydraulic jumps in both depth and stream width, flow separation, and two types of recirculations. The semigeostrophic prediction of the critical obstacle height proves accurate for relatively narrow channels and moderately accurate for wide channels. Significantly, we find that contact with the left-hand wall (facing downstream) is crucial to most of the interesting and important features. For example, no instances are found of hydraulic control of flow that is separated from the left-hand wall at the sill, despite the fact that such states have been predicted by previous semigeostrophic theories. The calculations result in a series of regime diagrams that should be very helpful for investigators who wish to gain insight into rotating, hydraulically driven flow.

\section{Introduction}

Robert Long's classical experiments, initially carried out in the 1950s (Long 1954) and later extended (Long 1970; Houghton \& Kasahara 1968; and Baines \& Davies 1980) form a cornerstone of hydraulic theory. In the original laboratory version of the experiment an obstacle is towed at a fixed speed through a channel of shallow fluid initially at rest. Numerical versions of the experiment place a fixed obstacle in the path of an initially steady, uniform flow, which is equivalent to the original set-up provided frictional effects are negligible. The outcome of the experiment for a single layer with a free surface depends on the Froude number $F_{0}=v_{0} / \sqrt{g d_{0}}$ based on 


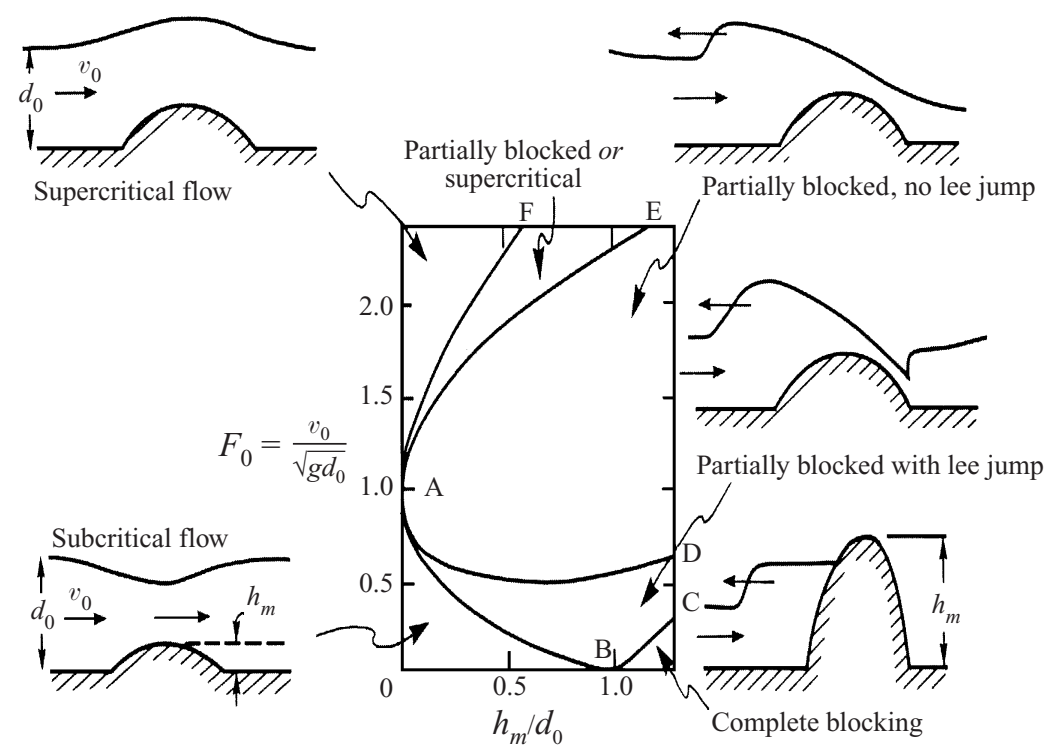

FIGURE 1. Regime diagram for Long's experiment with a homogeneous, shallow flow (Long 1954). The initial fluid depth is given by $d_{0}$, the obstacle height by $h_{m}$ and the towing speed by $v_{0}$. (Redrawn from Baines \& Davies 1980 figure 2.)

the initial depth $d_{0}$ and velocity $v_{0}$ of the moving stream and on a non-dimensional obstacle height $h_{m} / d_{0}$. Figure 1 shows the different steady regimes that develop over the obstacle and the transients that establish these regimes. For a given value of $F_{0}$ the outcome depends largely on whether $h_{m} / d_{0}$ exceeds a critical height (given by curve BAE). Beyond this height the obstacle partially blocks the approaching flow through the generation of a bore that moves upstream. The steady flow left behind has reduced volume transport and is hydraulically critical (hydraulically controlled) at the sill of the obstacle. For sufficiently large $h_{m} / d_{0}$ (given by the curve BC) the flow is completely blocked. Other boundaries can be calculated such as the curve AD, which separates flow having hydraulic jumps on the downslope of the obstacle from those that do not. The derivation of the curves forming the regime boundaries and examples of the development of the various flows within appear in Baines (1995, chap. 2).

Diagrams such as figure 1 and its generalizations in multi-layered flow are wonderful tools for developing knowledge and intuition about hydraulic jumps, bores, upstream influence, hydraulic control, and hydraulics in general. Naturally, a similar experiment carried out in a rotating channel would be of great use to investigators of deep strait and sill flow in the ocean, where the Coriolis effects can be important. A number of steady theories describing such flows exist, including benchmark papers by Whitehead, Leetmaa \& Knox (1974) and Gill (1977) and further studies by Borenäs \& Lundberg (1986), Pratt \& Armi (1987, 1991), Baines \& Leonard (1989), Dalziel (1990), Killworth (1992, 1994, 1995), Killworth \& McDonald (1993), Borenäs \& Whitehead (1998), Pratt \& Chechelnitsky (1998) and others reviewed by Pratt \& Lundberg (1991). As valuable as they have proven to be, such theories lack information about how hydraulically controlled flows are established, how upstream influence is exercised, how rotation influences bores and hydraulic jumps, and whether traditional shock joining can be performed across rotating jumps and bores. Also, laboratory experiments on rotating sill flows (Shen 1981 and Pratt 1987) have raised doubts about whether certain types of predicted flows can actually be realized. Unfortunately, performing 
something like Long's experiment in a rotating laboratory channel is technically difficult. In addition, traditional numerical models have been unable to handle all of the special problems, including separation of the stream from the channel sidewalls and shock formation, that inevitably occur in interesting regions of parameter space. Pratt (1983) was successful in obtaining some numerical solutions, but the range of parameters covered was severely limited by numerical difficulties. However, Helfrich, Kuo \& Pratt (1999) recently presented a code based on a numerical scheme used by Schär \& Smith (1993) that overcomes the difficulties just described. Our work has been made possible by development of this code.

The aim of this work has been to carry out numerical simulations of Long's experiment in a rotating channel. The presentation of these results is framed around prediction of the final steady flow based on the semigeostrophic approximation. The development of this approximation for shallow-water flow and the resulting constraints are reviewed in the next section. The semigeostrophic, shallow-water equations are then used $(\S 3)$ to solve for the threshold obstacle heights required to exert upstream influence (analogous to BAE of figure 1) or to induce flow separation from the channel sidewalls. In $\S 4$ we present numerical solutions based on the full shallow-water equations describing the time-dependent adjustment and the final steady state in the vicinity of the obstacle.

Aside from the insight we hope the reader will gain from browsing through our regime diagrams, the numerical solutions provide a vehicle for examining a number of long-standing mysteries concerning the hydraulics of rotating channel flow ( $\$ \$ 4$ and 5). To start with, we are able to explore the possibility of hydraulic control of a flow that is completely separated from one of the sidewalls of the channel. This situation is predicted by steady theory but has been difficult to reproduce in the laboratory. Although we create conditions favourable for hydraulically critical sill flow to occur, the subsequent sill flows revert to an attached state. This result suggests that it is difficult, if not impossible, for a separated flow to be hydraulically critical. Also we discover and map out different forms of shocks, rarefactions, and recirculations over much broader parameter ranges than have been possible in the past. An important new type of shock is the transverse hydraulic jump, a stationary and abrupt change in width of the stream. This was observed in a laboratory experiment by Pratt (1987) but has not been the subject of any further verification or study. The jump occurs where a separated and hydraulically supercritical flow abruptly widens to a subcritical and attached state. We discuss the momentum balance for this jump and investigate the possibility of performing shock-joining calculations. Along these lines, we also create conditions favourable for the formation of a moving transverse shock, in which the width of separated current suddenly increases from one value to another. A family of shocks having this property was predicted by Nof (1984) and we find similar examples. Another novel feature that arises in the numerical solutions is a rarefying intrusion that forms when a separated stream running along a sidewall collides with an obstacle of sufficient height. Some of the fluid passes over the obstacle and some is diverted to the opposing sidewall forming an intrusion. The result is a new mechanism for upstream influence. In addition to these interesting features, the numerical solutions allow us to identify breakdowns in uniform potential vorticity and/or semigeostrophic theory, the bases for most models of deep overflows. $\dagger$

$\dagger$ But not all: Pratt \& Armi (1987) and Killworth (1992) developed models for sill flow with non-uniform potential vorticity. Also, Killworth $(1994,1995)$ and Killworth \& McDonald (1993) have developed bounds governing the transport of deep overflows of arbitrary potential vorticity. 

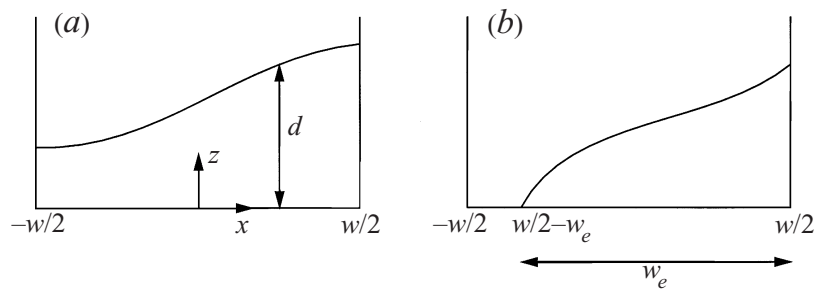

(c)

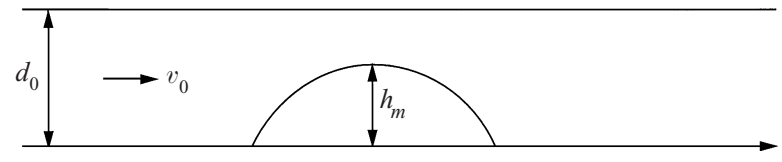

FIGURE 2. Definition sketch of rotating channel flow showing $(a)$ attached flow, (b) separated flow, (c) side view.

\section{Predictions based on semigeostrophic theory}

Consider a channel of rectangular cross-section, aligned in the $y$-direction, rotating at constant angular speed $f / 2$, and containing a shallow, inviscid, homogeneous fluid. The fluid may have finite depth across the entire channel, as shown in figure 2(a) or may be separated from one of the sidewalls, as in figure 2(b). Motion within the fluid is assumed hydrostatic and therefore governed by the two-dimensional shallow-water equations:

$$
\begin{gathered}
\frac{\partial u}{\partial t}+u \frac{\partial u}{\partial x}+v \frac{\partial u}{\partial y}-f v=-g \frac{\partial d}{\partial x}, \\
\frac{\partial v}{\partial t}+u \frac{\partial v}{\partial x}+v \frac{\partial v}{\partial y}+f u=-g \frac{\partial d}{\partial y}-g \frac{\partial h}{\partial y}, \\
\frac{\partial d}{\partial t}+\frac{\partial(u d)}{\partial x}+\frac{\partial(v d)}{\partial y}=0 .
\end{gathered}
$$

The coordinate system and variable definitions are given in figure $2(a-c)$.

From (2.1)-(2.3) it follows that the potential vorticity

$$
\frac{f+\partial v / \partial x-\partial u / \partial y}{d}
$$

is conserved following fluid columns. The potential vorticity is sometimes denoted $f / D_{\infty}$, where $D_{\infty}$, the potential depth, is the thickness to which a fluid column with a particular potential vorticity would have to be stretched in order to remove its relative vorticity $(\partial v / \partial x-\partial u / \partial y)$. We will deal only with flow having initially uniform potential vorticity, and therefore uniform $D_{\infty}$. This assumption is desirable from the standpoint of making comparisons with earlier rotating hydraulic theories (e.g. Whitehead et al. 1974 and Gill 1977), nearly all of which are based on the same assumption. In addition, the restriction limits the range of (already complex) behaviour and number of corresponding parameters. For example, uniform potential vorticity flows are known to have a boundary layer structure, the boundary layer thickness being given by $L_{d}=\sqrt{g D_{\infty}} / f$, the Rossby radius of deformation based on $D_{\infty}$. This scale is independent of the local depth and may therefore be taken as the only horizontal length scale, aside from those imposed by geometry. Further, the restriction to uniform $D_{\infty}$ has the important consequence of eliminating potential vorticity waves from the flow, at least in the early stages of evolution. Although non-uniformities in $D_{\infty}$ 
can develop due to shock formation or due to the weak dissipation present in the model, we observe no effects that could be attributed to potential vorticity waves. In particular, the critical control due to Rossby waves (discussed by Haynes, Johnson \& Hurst 1993 and Pratt \& Armi 1987) does not arise.

In what follows, $d$ and $h$ will be non-dimensionalized by $D_{\infty}, x$ by $L_{d}, v$ by $\left(g D_{\infty}\right)^{1 / 2}$, $y$ by the along-channel length scale $L$ imposed by the topography, and time $t$ by $L\left(g D_{\infty}\right)^{-1 / 2}$. Use of these scales in (2.3) then suggests scaling of $u$ by $\left(g D_{\infty}\right)^{1 / 2}\left(L_{d} / L\right)$. If $L$ is significantly larger than the cross-channel scale $L_{d}$, so that the horizontal aspect ratio $\delta=L_{d} / L \ll 1$, then the flow is nearly parallel $(u \ll v)$ and, generally speaking, $\partial / \partial x \gg \partial / \partial y$. Under these conditions the $y$-velocity is nearly in geostrophic balance:

$$
v=\frac{\partial d}{\partial x}
$$

where we now regard $v, d, x$, etc. as non-dimensional variables subject to the above scaling. The longitudinal velocity $u$ is generally not geostrophic and we therefore refer to $L_{d} / L \rightarrow 0$ as the 'semigeostrophic' limit. Also, the potential vorticity in this limit is

$$
\frac{1+\partial v / \partial x}{d}=1 \text {. }
$$

Our predictions of threshold obstacle heights are based on these simplifications.

The cross-sectional structure of the semigeostrophic flow can be determined from

$$
\frac{\partial^{2} d}{\partial x^{2}}-d=-1,
$$

obtained by eliminating $v$ from (2.5) and (2.6). For the present, assume that the flow is attached, meaning that $d>0$ across the entire channel width $-w / 2 \leqslant x \leqslant w / 2$. Following Gill (1977) a convenient representation of the solution of (2.7) is then

$$
d(x, y, t)=1+\hat{d}(y, t) \frac{\sinh (x)}{\sinh \left(\frac{1}{2} w\right)}+(\bar{d}(y, t)-1) \frac{\cosh (x)}{\cosh \left(\frac{1}{2} w\right)},
$$

and the associated geostrophic velocity is

$$
v(x, y, t)=\hat{d}(y, t) \frac{\cosh (x)}{\sinh \left(\frac{1}{2} w\right)}+(\bar{d}(y, t)-1) \frac{\sinh (x)}{\cosh \left(\frac{1}{2} w\right)} .
$$

In these expressions, the quantities $\hat{d}$ and $\bar{d}$ represent half the difference and sum, respectively, of the depth along the sidewalls:

$$
\bar{d}=\frac{1}{2}\left[d\left(\frac{1}{2} w, y, t\right)+d\left(-\frac{1}{2} w, y, t\right)\right]
$$

and

$$
\hat{d}=\frac{1}{2}\left[d\left(\frac{1}{2} w, y, t\right)-d\left(-\frac{1}{2} w, y, t\right)\right] .
$$

Using (2.9) these can also be related to the average and difference of the wall velocities:

$$
\bar{v}=\frac{1}{2}\left[v\left(\frac{1}{2} w, y, t\right)+v\left(-\frac{1}{2} w, y, t\right)\right]=T^{-1} \hat{d}
$$

and

where

$$
\hat{v}=\frac{1}{2}\left[v\left(\frac{1}{2} w, y, t\right)-v\left(-\frac{1}{2} w, y, t\right)\right]=T(\bar{d}-1),
$$

$$
T=\tanh \left(\frac{1}{2} w\right) .
$$

A consequence of semigeostrophy, first recognized by Gill (1977), is that separation 
of the flow caused by the vanishing of $d$ cannot occur at an interior point of the stream. Suppose that $\left(x_{0}, y_{0}\right)$ is the first such point for this vanishing to occur, so that $d\left(x_{0}, y_{0}\right)=0$ but $d>0$ in a small $x$-neighbourhood of $\left(x_{0}, y_{0}\right)$. Then, provided that $d$ is twice differentiable with respect to $x,\left(\partial^{2} d / \partial x^{2}\right)_{\left(x_{0}, y_{0}\right)}>0$ in violation of (2.7). One consequence is that the vanishing of $d$ at a section where $d$ is initially non-zero all across the channel must occur first along a sidewall. We will refer to the subsequent detachment of the flow from the sidewall, and formation of a free edge, as flow separation. This type of separation should be distinguished from the streamline separation coincident with a stagnation point along a wall in the presence of non-zero depth.

In the limit of strong rotation $(w \rightarrow \infty),(2.8)$ becomes

$$
d=1+[d(w / 2, y, t)-1] \mathrm{e}^{(x-w / 2)}+[d(-w / 2, y, t)-1] \mathrm{e}^{-(w / 2+x)}+O\left(\mathrm{e}^{-w}\right)
$$

and thus the solution takes on a boundary layer structure with the sidewall depths decaying to the interior value (unity) over the Rossby radius of deformation (also unity).

The evolution of a semigeostrophic flow from given initial conditions may be calculated by substituting (2.8) and (2.9) into (2.1) and (2.3) and solving the resulting hyperbolic equations for the coefficients $\bar{d}(y, t)$ and $\hat{d}(y, t)$ using the method of characteristics. As shown by Pratt (1983), the characteristic speeds are given by

$$
c_{ \pm}=T^{-1} \hat{d} \pm \bar{d}^{1 / 2}\left[1-T^{2}(1-\bar{d})\right]^{1 / 2} .
$$

Accordingly, a Froude number

$$
F_{d}=\frac{T^{-1} \hat{d}}{\bar{d}^{1 / 2}\left[1-T^{2}(1-\bar{d})\right]^{1 / 2}}
$$

may be defined such that the flow is locally subcritical for $F_{d}<1$ (implying that signals can propagate in both $\pm y$-directions), supercritical for $F_{d}>1$ (implying that both signals propagate in the same direction), and critical for $F_{d}=1$ (implying the speed of one of the modes is zero).

In the limit of weak rotation, $w \rightarrow 0$ (or $T \rightarrow 0$ ), $c_{+}$reduce to the familiar long gravity wave speeds $v \pm d^{1 / 2}$. In the opposite limit, $w \rightarrow \infty$ (or $T \rightarrow 1$ ), these speeds become

and

$$
c_{+}=v(w / 2, y, t)+1=d(w / 2, y, t)
$$

$$
c_{-}=v(-w / 2, y, t)-1=-d(-w / 2, y, t) .
$$

As suggested by (2.14) the waves have now separated into two independent Kelvin waves, each trapped against a sidewall. For the wave trapped on the wall at $x=w / 2$, which will be called the right-hand wall, the characteristic speed is positive provided the depth at that wall is non-zero (the flow is attached). Similarly, the left-hand wall $(x=-w / 2)$ wave has negative speed for non-zero left-hand wall depth. All flows of finite depth in infinitely wide channels are therefore subcritical in the sense that the two long waves propagate in opposite directions. In order for critical flow to form it is necessary for the flow to separate from one of the walls or for the channel width to be less than several $L_{d}$, forcing the Kelvin waves to overlap.

When the flow separates from one of the sidewalls, the above expressions for the wave speeds remain valid but the character of the waves is altered. The Kelvin waves, which have zero $u$ in the limit of small amplitude, become frontal waves, which have 
finite $u$ (Stern 1980). In addition, the dependent variables change from $\bar{d}$ and $\hat{d}$ (which become equal for separated flow) to $\bar{d}$ and $w_{e}$, the latter denoting the separated width of the current (figure $2 b$ ).

For steady, semigeostrophic flow with uniform potential vorticity, solutions for flow passing over obstacles or through contractions in the channel width can be computed from a known upstream condition by conserving volume flux and energy (Bernoulli function). A common form of the conserved energy is the average $\bar{B}$ of the semigeostrophic Bernoulli functions $v^{2} / 2+d+h$ on the two sidewalls, or

$$
\bar{B}=\frac{1}{2}\left[T^{-2} \hat{d}^{2}+T^{2}(\bar{d}-1)^{2}\right]+\bar{d}+h
$$

in view of (2.12) and (2.13). In addition, the volume transport $Q=\int_{-w / 2}^{w / 2}(v d) \mathrm{d} x$ must be conserved, and it can be shown using (2.5), (2.10), and (2.11) that

$$
Q=2 \bar{d} \hat{d} \text {. }
$$

For detached flow the equations governing steady flow can be obtained by replacing $\hat{d}$ by $\bar{d}$ and $T$ by $T_{e}=\tanh \left(w_{e} / 2\right)$ in (2.19) and (2.20).

\section{Initial conditions and the critical obstacle height}

\subsection{Parameters and initial conditions}

A number of complications are introduced in the rotating version of Long's experiment. First, towing an obstacle along the channel at a fixed speed through an initially stationary fluid is no longer equivalent to introducing a stationary obstacle in a moving stream of the same speed. In the first case the free surface is horizontal; in the second it has a cross-stream, geostrophic tilt. We have chosen to perform the second version of the experiment as the upstream states seem more meaningful for ocean applications. Thus, the obstacle will be introduced into a steady current that is uniform in $y$ but that varies with $x$. This current will have uniform potential vorticity and will thus have the form given by the velocity and depth profiles (2.8) and (2.9).

A second complication is the introduction of three new length scales $\left(L_{d}, D_{\infty}\right.$, and the channel width), leading to two additional dimensionless parameters. There are now four parameters in all and these may be picked in a variety of ways. To maintain continuity with Long's original experiment, we continue to use the Froude number of the initial flow, defined by (2.16), and the dimensionless obstacle height $h_{m}$. In addition, we select $w$ (the dimensional channel width divided by $L_{d}$ ), which determines the overall importance of rotation. A fourth parameter could also be introduced, but this would lead to an overwhelmingly large parameter space to explore. In Gill's (1977) formulation the fourth parameter (his $\psi_{i}$ ) governs the relative amounts of volume flux contained in the right- and left-hand wall boundary layers. As a starting point, we will fix the fourth parameter by requiring that the total volume flux of the initial flow is contained in the left-hand wall boundary layer. This assumption can be motivated by imagining that the initial flow has been set up as the result of a dam break experiment in which motion is triggered by a Kelvin wave propagating upstream along the left-hand wall. This situation is now described in more detail.

Suppose that the initial stream in which the obstacle is to be introduced has been set up by breaking a dam, as suggested in figure 3. The dam lies at $y=0$ and the initial depth is zero for $y>0$. Behind the dam $(y<0)$ lies fluid that is at rest and that therefore has dimensional depth $D_{\infty}$. Far from the dam in the negative $y$-direction, the channel broadens into wide reservoir. If the dam is destroyed, a positive flow is set 
(a)

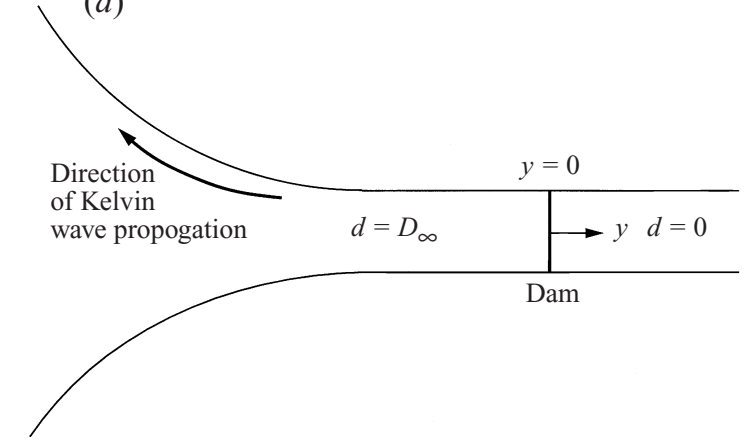

(b)

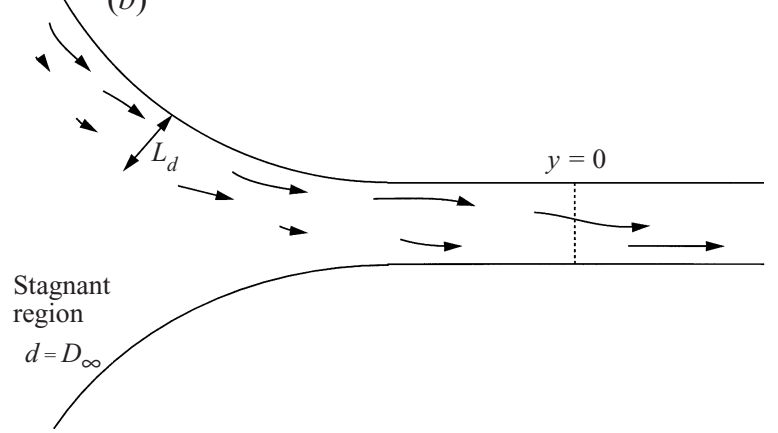

FIGURE 3. Sketch illustrating the establishment of the flow in the channel after a dam break. The final steady current is lies along the 'left' wall in the infinite upstream reservoir. (a) Before dam break, $(b)$ after dam break.

up in $y<0$ by a Kelvin wave that moves away from the original dam (Helfrich et al. 1999). Upon entering the wide reservoir, this wave becomes trapped to the left-hand wall (facing downstream) as indicated in figure 3(b). Hence, the current emanating from the reservoir is trapped within a distance $L_{d}$ of this wall and the fluid near the right-hand wall in the reservoir remains stagnant. In the narrower section of channel near the dam, moving fluid may exist across the entire width. Although the reservoir will not appear explicitly in our numerical calculations, we will imagine that it exists far upstream of the boundaries of our numerical domain, and that the initial flow emanating from the reservoir is trapped to the left-hand wall. $\dagger$ We now show how this assumption allows reduction of the number of independent parameters from four to three $\left(F_{d}, h_{m}\right.$, and $\left.w\right)$.

The procedure for specializing the initial conditions to give zero approach flow along the right-hand wall of the hypothetical reservoir is based on conservation of energy along that wall. Since the flow along the reservoir's right-hand wall is stagnant,

$\dagger$ When the initial flow is attached to both sidewalls, a gradual upstream widening of the channel will result in diversion of all the volume transport to the left-hand boundary layer. When the initial flow is separated from the left-hand wall it can be shown, however, that the current will remain separated as the channel widens. In this case, a combination of width and bottom elevation changes, with possible hydraulic transitions, may be necessary to provide a link to the hypothetical reservoir. 
the value of the Bernoulli function there is unity (dimensionally $g D_{\infty}$ ), and thus

$$
\frac{v_{0}^{2}(w / 2)}{2}+d_{0}(w / 2)=1,
$$

where the ()$_{0}$ denotes initial values. If $(2.10)-(2.13)$ are used to write this relation in terms of $\bar{d}$ and $\hat{d}$ the result may be expressed in the non-dimensional form

$$
\frac{\left[\left(\hat{d}_{0} / T\right)-T\left(1-\bar{d}_{0}\right)\right]^{2}}{2}+\bar{d}_{0}+\hat{d}_{0}=1
$$

To fix the initial conditions for given $F_{d}$ and $w$ the values of $\bar{d}_{0}$ and $\hat{d}_{0}$ must be computed. Once known, these two quantities completely determine the initial depth and velocity profiles through (2.8) and (2.9). Equation (3.1a) provides one equation for $\bar{d}_{0}$ and $\hat{d}_{0}$ while $(2.16)$ provides a second. If $\hat{d}_{0}$ is eliminated between these two relations the following equation for $\bar{d}_{0}$ is obtained:

$$
\frac{1}{2}\left\{F_{d} \bar{d}_{0}^{1 / 2}\left[1-T^{2}\left(1-\bar{d}_{0}\right)\right]^{1 / 2}-T\left(1-\bar{d}_{0}\right)\right\}^{2}+\bar{d}_{0}+F_{d} T \bar{d}_{0}^{1 / 2}\left[1-T^{2}\left(1-\bar{d}_{0}\right)\right]^{1 / 2}=1 .
$$

It may turn out that the initial flow is separated from the left-hand wall of the channel, in which case the above calculation will give $\bar{d}_{0}<\hat{d}_{0}$. In this situation, the parameter $T$ in $(3.1 b)$ must be replaced by the variable $T_{e 0}=\tanh \left(w_{e 0} / 2\right)$, where $w_{e 0}$ is the initial width of the separated current. The initial condition is now specified by the value of $\bar{d}_{0}$ (now equal to $\hat{d}_{0}$ ) and $T_{e 0}$, and both are related by

$$
\bar{d}_{0}=\frac{F_{d}^{2}\left(1-T_{e 0}^{2}\right)}{T_{e 0}^{-2}-F_{d}^{2} T_{e 0}^{2}}
$$

which follows from (2.16). Substitution of this relation into (3.1b) results, after some rearrangement, in

$$
\frac{\left(F_{d}^{2}-1\right)^{2}}{2}+\left(1-F_{d}^{2} T_{e 0}^{4}\right)\left(2 F_{d}^{2}-F_{d}^{2} T_{e 0}^{2}-T_{e 0}^{-2}\right)=0 .
$$

The procedure is to first solve $(3.2 b)$ for $T_{e 0}$ and then calculate the corresponding value of $\bar{d}_{0}$ from $(3.2 a)$.

One consequence of the assumption that the volume flux in the initial flow is fed from the reservoir's left-hand boundary layer is that separated initial flow cannot be subcritical. To prove this result, write $(3.2 b)$ in the form $\frac{1}{2}\left(1-F_{d}^{2}\right)^{2}+a b=0$, where $a=\left(1-F_{d}^{2} T_{e 0}^{4}\right)$ and $b=\left(2 F_{d}^{2}-F_{d}^{2} T_{e 0}^{2}-T_{e 0}^{-2}\right)$, and suppose that the initial flow is subcritical $\left(F_{d}^{2}<1\right)$. Then as $T_{e 0}^{2}$ varies over its possible range $[0,1] a$ varies monotonically from unity to $\left(1-F_{d}^{2}\right)$, whereas $b$ varies monotonically from $-\infty$ to $\left(F_{d}^{2}-1\right)$, so that $a b$ varies from $-\infty$ to $-\left(1-F_{d}^{2}\right)^{2}$. Hence $a b$ does not cross the value $-\frac{1}{2}\left(1-F_{d}^{2}\right)^{2}$ required to satisfy equation $(3.2 b)$.

\subsection{The critical obstacle height}

It is anticipated that only values of $h_{m}$ greater than some critical value $h_{c}$ will lead to upstream influence: permanent alteration of the upstream flow. In Long's experiment the physical argument on which $h_{c}$ is based involves the transport $Q$ and energy (Bernoulli constant) $B$ of the initial flow. Imagine a steady flow passing over an obstacle of height $h_{m}$ and having the same values of $Q$ and $B$ as the initial state under consideration. Then there is a maximum $h_{m}$ for which this flow has sufficient energy $B$ (at the given $Q$ ) to surmount the crest. It can be shown from steady hydraulic theory that this maximum $h_{m}$ is also the height required to render the sill flow critical. If 
$h_{m}$ exceeds the maximum allowable value, the values of $B$ and/or $Q$ must be altered in order to allow the flow to continue and this implies generation of an upstream disturbance that alters the values of $Q$ and $B$. Thus, the predicted $h_{c}$ for given initial $Q$ and $B$ is that height for which $Q$ and $B$ would, in a steady state, produce critical sill flow. An application of the same principles (with the upstream state now specified by $F_{d}$ and $w$ ) results in a prediction of $h_{c}$ in the rotating case.

Consider a steady flow with upstream values $\bar{d}=\bar{d}_{0}$ and $\hat{d}=\hat{d}_{0}$ that becomes critical $\left(\bar{d}=\bar{d}_{c}\right.$ and $\left.\hat{d}=\hat{d}_{c}\right)$ at the crest $\left(h=h_{c}\right)$ of the obstacle. (Note that $\bar{d}_{c}$ and $\hat{d}_{c}$ play a similar role to $Q$ and $B$ in the example of the previous paragraph.) Conservation of mass (2.20) requires that

$$
\hat{d}_{0} \bar{d}_{0}=\hat{d}_{c} \bar{d}_{c} .
$$

Together with the condition of criticality at the sill $\left(F_{d}=1\right.$ in 2.16), (3.3) implies that

$$
\bar{d}_{c}^{4}+\frac{1-T^{2}}{T^{2}} \bar{d}_{c}^{3}-T^{-4}\left(\hat{d}_{0} \bar{d}_{0}\right)^{2}=0 .
$$

This equation determines $\bar{d}_{c}$ given the upstream/initial quantities $\hat{d}_{0}$ and $\bar{d}_{0}$. The value of $\hat{d}_{c}$ then follows from (3.3). Once $\bar{d}_{c}$ and $\hat{d}_{c}$ have been found it must be determined whether or not the flow at the sill is separated. If $\bar{d}_{c} \geqslant \hat{d}_{c}$ the flow is non-separated and one may proceed to the next step, as described below. If $\bar{d}_{c}<\hat{d}_{c}$ the flow at the sill is separated from the left-hand wall, and (3.4a) must be revised by setting $\bar{d}_{c}=\hat{d}_{c}=\left(\hat{d}_{0} \bar{d}_{0}\right)^{1 / 2}$ and $T=T_{e c}=\tanh \left(w_{e c} / 2\right)$, where $w_{e c}$ is the separated width of the flow at the sill. Substitution into (3.4a) then yields a formula for $T_{e c}$ :

$$
T_{e c}^{2}=\frac{\left(\hat{d}_{0} \bar{d}_{0}\right)^{1 / 2}}{1-\left(\hat{d}_{0} \bar{d}_{0}\right)^{1 / 2}} .
$$

In either case the properties of the critical flow at the sill are known.

The critical sill height $h_{c}$ can now be computed by equating the energy at the sill with that upstream. Employing the Bernoulli equation (2.19) with the computed values of $\hat{d}_{c}$ and $\bar{d}_{c}$ leads, in the case of non-separated flow, to

$$
h_{m}=1-\hat{d}_{0} \bar{d}_{0}-\frac{1}{2}\left[T^{2}\left(\bar{d}_{c}-1\right)^{2}+\left(\hat{d}_{c} / T\right)^{2}+2 \bar{d}_{c}\right] .
$$

When the sill flow is separated, this relation is replaced by

$$
h_{m}=1-\hat{d}_{0} \bar{d}_{0}-\frac{1}{2}\left\{T_{e c}^{2}\left[\left(\hat{d}_{0} \bar{d}_{0}\right)^{1 / 2}-1\right]^{2}+\frac{\hat{d}_{0} \bar{d}_{0}}{T_{e c}^{2}}+2\left(\hat{d}_{0} \bar{d}_{0}\right)^{1 / 2}\right\}
$$

with the value of $T_{e c}$ from (3.4b).

Figure 4 shows a plot of $h_{m}$ as a function of $F_{d}$ for a case of weak rotation $(w=0.5)$. The relationship is given by the curve CAE, which is composed of a number of segments indicating various states of separation. To the left of CAE, there is no predicted upstream influence and the final flow upstream and downstream of the obstacle is identical to the initial flow. Over the obstacle the final flow is disturbed but does not become critical. To the right of CAE, the predicted final upstream and downstream states have been altered by (unknown) transients. The predicted flow over the obstacle is critical at the sill and supercritical in the lee, possibly with some form of hydraulic jump. On CAE, the predicted flow is critical at the obstacle crest but the upstream flow is unaltered. Along the solid segment $B A^{\prime}$, both the initial flow and the predicted sill flow are non-separated. Along $\mathrm{BC}$, which lies at the extreme lower right of the diagram, and is enlarged in an inset, the initial flow is attached 


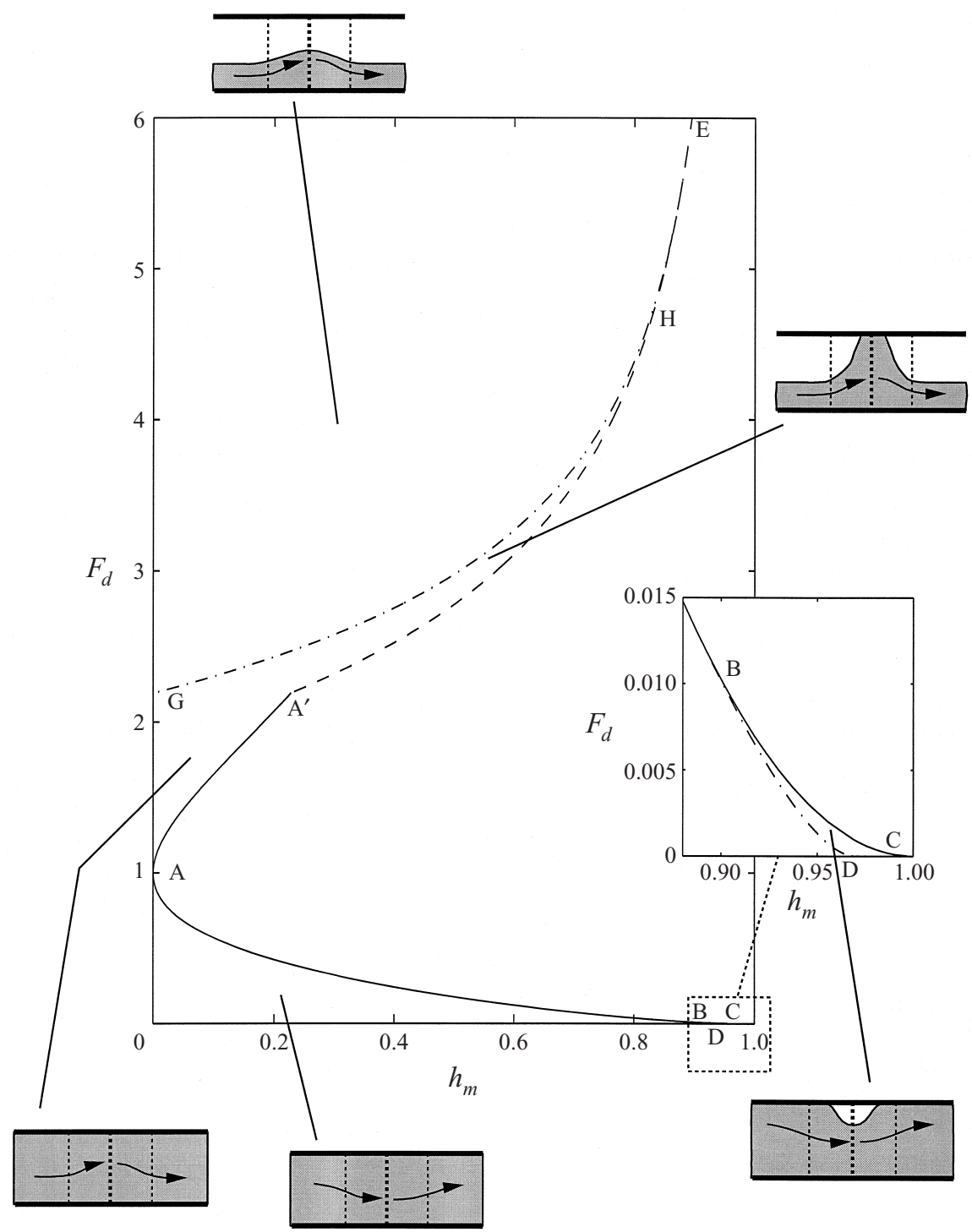

FIGURE 4. Regime diagram showing the predicted response in terms of the initial Froude number $F_{d}$ and the obstacle height $h_{m}$, all for a channel of width $w=0.5$. The curve CAE gives the critical obstacle height, with different segments indicating different states of flow separation. The curves DB and GH indicate various states of flow separation for completely subcritical or supercritical flows. See the text for more details.

but the predicted critical sill flow is separated. The predicted final flow thus separates from the left-hand wall at some point slightly upstream of the sill. To the immediate left of $\mathrm{BC}$ the upstream flow is attached and subcritical and the predicted flow over the obstacle is also subcritical but detached at the sill. In the upper portion of the diagram corresponding to supercritical initial flow $\left(F_{d}>1\right)$ lies a segment $\mathrm{A}^{\prime} \mathrm{E}$ spanning a range of Froude numbers for which the initial flow is separated. Along sub-segment $\mathrm{A}^{\prime} \mathrm{H}$ the predicted sill flow is critical and attached while along $\mathrm{HE}$ the sill flow is critical and separated. To the immediate left of $\mathrm{A}^{\prime} \mathrm{H}$ lies a wedge-shaped region $\mathrm{A}^{\prime} \mathrm{HG}$ in which the predicted final flow is supercritical everywhere, separated 


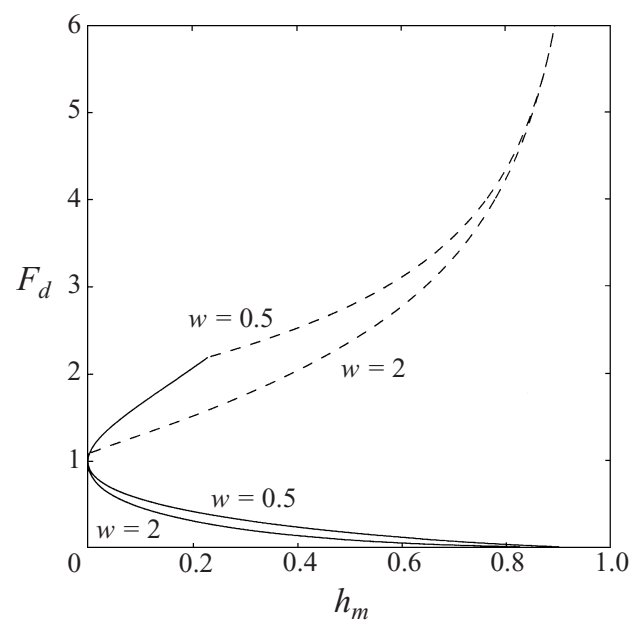

Figure 5. Curves of critical obstacle height $h_{m}=h_{c}$ as a function of $F_{d}$ for $w=0.5$ and $w=2$.

upstream and downstream of the obstacle, and attached near the sill. To the left of HE the predicted final flow is supercritical and separated everywhere.

An idea of the influence of rotation on the critical obstacle height can be gained by inspection of figure 5, which shows the critical height curve CAE from the above weak rotation case $(w=0.5)$ plotted along with the $w=2$ relation. For subcritical initial conditions rotation reduces the critical obstacle height whereas the reverse is true when the initial flow is supercritical. Note that the two curves merge when $F_{d}$ is sufficiently large. Here the initial flow and the predicted sill flow are separated, implying that $w$ is no longer a factor in determining $h_{m}$.

\subsection{Marginal separation at the sill}

Along segment $\mathrm{BC}$ of figure 4 the initial flow is attached and the predicted critical sill flow separated. Reducing the sill height but keeping $F_{d}$ fixed should eventually result in a flow that is attached everywhere (and subcritical everywhere). The value of $h_{m}$ at which the sill flow is marginally seperated (i.e. $d_{s}(-w / 2)=0$ and $w=w_{e}$ ) will be denoted $h_{s}$. For $h_{c} \geqslant h \geqslant h_{s}$ the predicted sill flow is subcritical but separated; for $h<h_{s}$ the predicted sill flow is subcritical and attached. $\dagger$ In either case, the solutions are not hydraulically controlled and have upstream/downstream symmetry relative to the sill. The value of $h_{s}$ can be calculated by replacing the critical condition in the steps leading to (3.5a) by the condition of marginal separation $\left(\bar{d}=\hat{d}\right.$ and $\left.T_{e}=T\right)$ at the sill. This procedure results in

$$
h_{s}=\frac{1}{2} T^{2}\left[\left(\bar{d}_{0}-1\right)^{2}-\left(\bar{d}_{s}-1\right)^{2}\right]+\frac{\hat{d}_{0}^{2}-\bar{d}_{s}^{2}}{2 T^{2}}+\bar{d}_{0}-\bar{d}_{s},
$$

where $\bar{d}_{s}=\hat{d}_{s}=\left(\bar{d}_{0} \hat{d}_{0}\right)^{1 / 2}$. The curve of $h_{s}$ vs. $F_{d}$ is given by DB in the inset at the right of figure 4 . To the left of this curve the predicted flow is subcritical and attached everywhere. In the region DBC, the flow is separated at the sill, attached upstream and downstream of the obstacle, and subcritical everywhere.

$\dagger$ There may also exist a curve of marginal separation to the right of $\mathrm{BAA}^{\prime}$ corresponding to a hydraulically controlled solution with marginal separation at the sill. However, the corresponding curve cannot be calculated without some knowledge of the upstream disturbance that sets up the controlled flow. 
Similarly, there exists a range of supercritical initial conditions for which the predicted final states are supercritical everywhere, separated upstream and downstream of the sill, and marginally separated at the sill. As before, the obstacle height $h_{s}$ for such solutions is smaller than the corresponding critical height and can be calculated by replacing the critical condition by the condition of marginal separation, this time in the steps leading to $(3.5 b)$. This procedure yields

$$
h_{s}=\frac{1}{2}\left(T_{e 0}^{2}-T^{2}\right)\left(\bar{d}_{0}-1\right)^{2}+\frac{1}{2} \hat{d}_{0}^{2}\left(T_{e 0}^{-2}-T^{-2}\right) .
$$

The corresponding curve is labelled $\mathrm{GH}$ in figure 4. The region $\mathrm{GHA}^{\prime}$ contains flows which are supercritical everywhere, separated upstream and downstream of the obstacle, and attached at the sill, as shown by the inset. To the left of GE the predicted final flows are supercritical and separated everywhere.

\section{Temporal evolution}

We now describe a numerical exploration of the parameter space defined above using the full, two-dimensional, shallow water equations.

\subsection{Numerical method}

The numerical model used for this study is the same one described in Helfrich et al. (1999) in a study of the nonlinear Rossby adjustment problem in a rotating channel. The model solves the non-dimensional shallow water equations in flux form,

$$
\begin{gathered}
\frac{\partial}{\partial t}(u d)+\frac{\partial}{\partial x}\left(u^{2} d+\frac{1}{2} d^{2}\right)+\frac{\partial}{\partial y}(u v d)-v d+d \frac{\partial h}{\partial x}=\mu \nabla \cdot(d \nabla u), \\
\frac{\partial}{\partial t}(v d)+\frac{\partial}{\partial y}\left(v^{2} d+\frac{1}{2} d^{2}\right)+\frac{\partial}{\partial x}(u v d)+u d+d \frac{\partial h}{\partial y}=\mu \nabla \cdot(d \nabla v), \\
\frac{\partial d}{\partial t}+\frac{\partial}{\partial x}(u d)+\frac{\partial}{\partial y}(v d)=0 .
\end{gathered}
$$

The discrete forms of mass and momentum conservation are obeyed across discontinuities provided the topographic slope $\nabla h=0$. Note that the lateral dissipation terms on the right-hand sides of (4.1)-(4.3) are also in flux form. The frictional terms are not necessary for numerical stability but help to reduce dispersive oscillations (Gibb's phenomena) which occur in the neighbourhood of jumps.

The numerical method follows closely the technique introduced by Schär \& Smith (1993) for the non-rotating version of (4.1)-(4.3). The principal exception is the replacement of the MPDATA algorithm for the computation of the advective fluxes (Smolarkiewicz \& Clark 1986) with a more efficient slope-limited flux-corrected transport method described in LeVeque (1997). The numerical scheme is secondorder in space and time. The model permits the development and accurate evolution of shocks, bores and jumps. It also allows for zero layer depth (or nearly so, since layer depths are limited to a minimum of $10^{-10}$ ). The numerical model has been tested against analytical solutions for both shock propagation and the evolution of the contact line between wet and dry regions. More details of the model and testing are discussed in Helfrich et al. (1999).

The channel sidewalls at $x= \pm w / 2$ are treated with slip and no-flux conditions to keep the solutions close to the inviscid semigeostrophic theory. Orlanski (1976) radiation conditions are used at the channel ends. The numerical results presented in this paper use a uniform cell-centred grid with spacing in the along-channel direction 
of $\Delta y=0.05$. The cross-channel grid spacing and time steps range from $(\Delta x=0.025$ and $\Delta t=0.01)$ at $w=0.5$ to $(\Delta x=0.05$ and $\Delta t=0.02)$ at $w=2$ and 4 . Numerical convergence was tested by decreasing $\Delta x, \Delta y$ and $\Delta t$ by a factor of 2 for several test cases. Increased resolution results in slightly sharper resolution of discontinuities. However shock and jump locations and speeds are unaffected as are regions of vanishing layer depth. The viscous parameter is $\mu=0.005$, a value large enough to help smooth the solution near discontinuities without resulting in excessive lateral diffusion of momentum.

The experiments were started at $t=0$ with the flow specified in terms of $F_{d}$ and $w$ by the semigeostrophic solution (2.8), (2.9) as described in $\S 3.1$. A Gaussian topographic feature which varies in the along channel direction only,

$$
h=h_{0}(t) \exp \left(-\lambda^{2} y^{2}\right),
$$

is then grown into the flow. The amplitude $h_{0}(t)$ is increased linearly from zero to $h_{m}$ from $t=0$ to $t=t_{m}$ and is held fixed thereafter. The growth time scale is $t_{m}=2$ and the width scale is $\lambda=0.5$ for all experiments. The numerical domain is typically 40 or 50 units in the $y$-direction. In some cases the domain was extended in the $y$-direction to avoid problems associated with the radiation conditions on the upstream boundary.

\subsection{A tour of the numerical results}

The numerical results are summarized in figures 6,7 and 8 for channel widths $w=0.5,2$, and 4 , respectively. These widths were chosen because they enable us to investigate the variations from narrow to relatively wide channels. In these figures the regime curves from the semigeostrophic theory are shown along with the locations of numerical runs. The circles indicate solutions exhibiting a lack of permanent alteration of the original flow and the squares show cases exhibiting permanent upstream influence. The numerical results show versions of most of the features, including bores and jumps, that arise in Long's original experiments. They also reveal some features which are remarkable and unexpected. Since it is not possible to discuss each numerical run in detail, the reader is referred to the thumbnail insets in figures 6-8 showing characteristic behaviour found in different regions of the parameter space. These insets contain contours of the free-surface height, $d(x, y, t)+h(y)$, at later stages of the flow development. They illustrate the final steady flows over the topography and, in some cases, the structure of transient features. The grey shaded regions in some of the insets indicate areas of the channel which are 'dry', defined by $d<0.001$. This choice for the contact line is arbitrary, but the results are not sensitive to it. The dashed lines are contours of the bottom topography of $1,0.5$ and 0.001 times $h_{m}$.

The occurrence of upstream influence was defined by asymmetry in the alongchannel direction of the final steady flow over the topography and by a reduction in the transport at the sill crest compared to the initial transport. For subcritical initial flow $\left(F_{d}<1\right)$ the numerically determined transition to upstream influence agrees reasonably well with the semigeostrophic theory for $w=0.5$ and 2 (figures 6 and 7). For $w=4$ and small $F_{d}$ (figure 8) the numerical results indicate upstream influence for smaller values of $h_{m}$ than predicted by the theory. We did not attempt to determine the transition points with any finer detail than indicated since the effects on symmetry or transport are very small and take a long time to develop in the neighbourhood of the transition. Further, the presence of friction in the model will act to break the symmetry. The agreement extends to $F_{d}>1$ for the narrow channel $w=0.5$ (figure 6). For $w=2$ and 4 (figures 7 and 8) the transition to upstream influence occurs at moderately larger values of $h_{m}$ than predicted by the theory, though the 


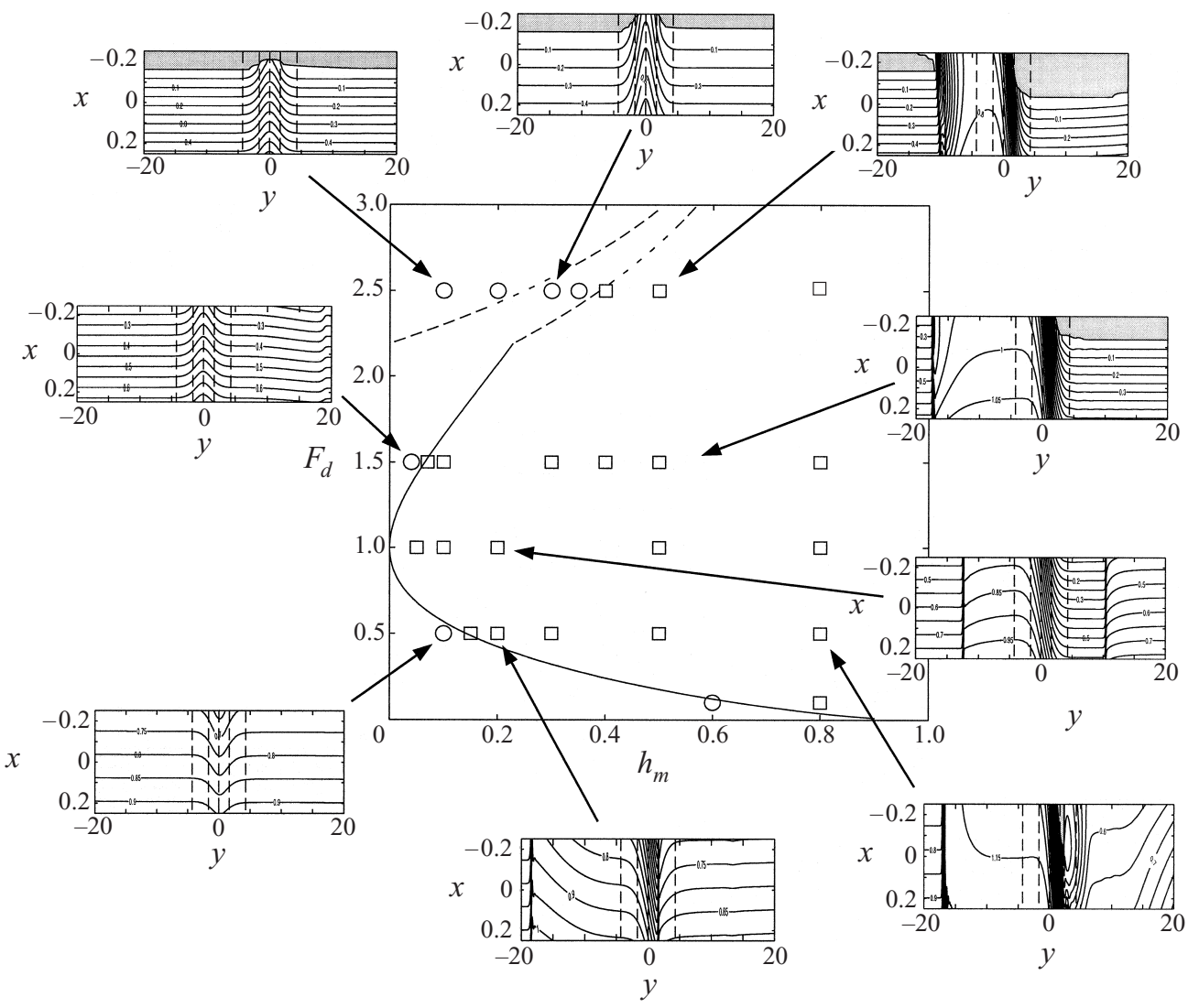

FIGURE 6. Summary of the numerical results for $w=0.5$. The regime curves from the semigeostrophic theory are shown along with the locations of numerical runs. The circles indicate no permanent alteration of the original flow and the squares show cases of permanent upstream influence. Also shown are inset examples of the numerical results. The insets show contours of the free-surface height $(d(x, y, t)+h(y))$. The shaded regions indicate those portions of the channel which are 'dry' (defined by $d<0.001$ ). The dashed lines are the $1,0.5$ and 0.001 times $h_{m}$ contours of the bottom topography.

general behaviour of the transition as a function of $F_{d}$ follows the theory for the few values of $F_{d}>1$ that have been investigated.

The disagreement between the numerically determined transitions and the semigeostrophic theory with increasing channel width is not surprising. In narrow channels the confinement provided by the walls suppresses cross-channel accelerations and thus the along-channel flow should remain nearly geostrophic as required for the semigeostrophic approximation to be valid. For wider channels this effect is weakened and large cross-channel accelerations occur over the sill in the initial adjustment phase leading to departure from the semigeostrophic prediction. These effects were also observed in the dam break problem studied by Helfrich et al. (1999). Nonconservation of potential vorticity could also affect the value of the critical height. The numerical model includes weak lateral viscosity and thus does not conserve potential vorticity following fluid parcels as the analytical model does. Fluid parcels which pass through shocks can also have their potential vorticity altered due to the implicit energy loss associated with the shock. 


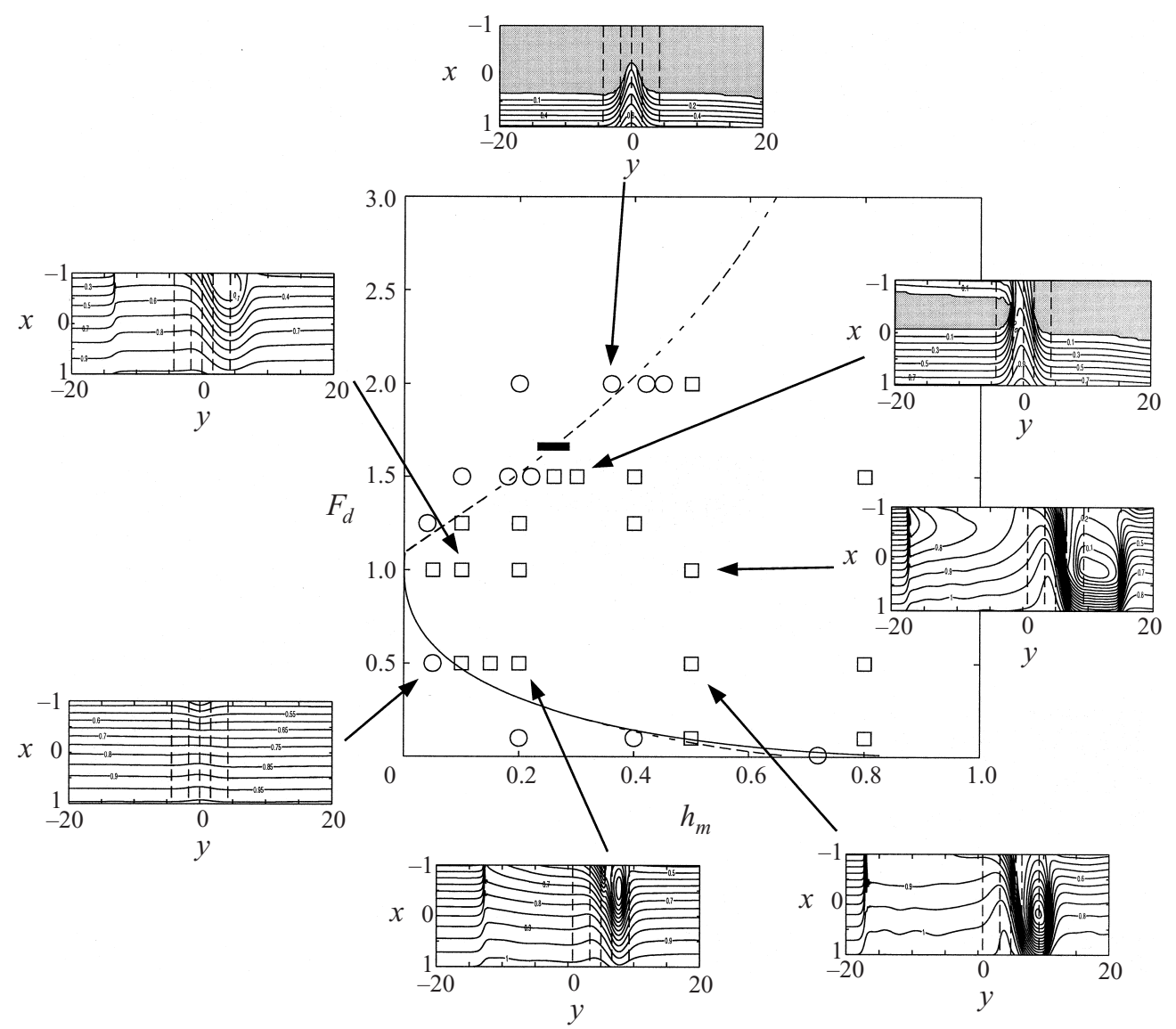

FiguRE 7. Same as figure 6 except $w=2$. Dashing of the critical obstacle height curve indicates those values of $F_{d}$ for which the initial flow is separated. The thick horizontal bar overlaid on the critical obstacle height curve indicates the value of $F_{d}$ above which the predicted critical sill flow is separated from the left wall.

\subsubsection{Case $w=0.5$}

We begin the discussion of the flow evolution by examing the case $w=0.5$, as summarized in figure 6. Despite the narrowness of this channel, rotation can be quite important. First, consider some examples for which there is no predicted upstream influence $\left(h_{m}<h_{c}\right)$ as illustrated by the insets on the left-hand side of figure 6 . Subcritical conditions give rise to an acceleration of the flow accompanied by a deflection of streamlines over the obstacle towards the right-hand wall (e.g. $F_{d}=0.5$, $\left.h_{m}=0.1\right)$. The opposite occurs for supercritical initial conditions, as exemplified by the case $\left(F_{d}=1.5, h_{m}=0.04\right)$. If $F_{d}$ is large enough the initial supercritical flow is separated and the corresponding final steady states may either be completely separated $\left(F_{d}=2.5, h_{m}=0.1\right)$ or separated away from but attached near the sill $\left(F_{d}=2.5, h_{m}=0.3\right)$. This last case is shown in greater detail in figure 9. At $t=10$ the disturbance generated by the introduction of the topography is evident immediately downstream of the sill. It consists of two waves which propagate downstream. The first is the faster Kelvin wave, centred at about $y=15$, and the second is the slower frontal wave (Stern 1980), centred at about $y=8$. Note that in linear wave dynamics the Kelvin wave should have no expression on the contact line. However, in this fully 


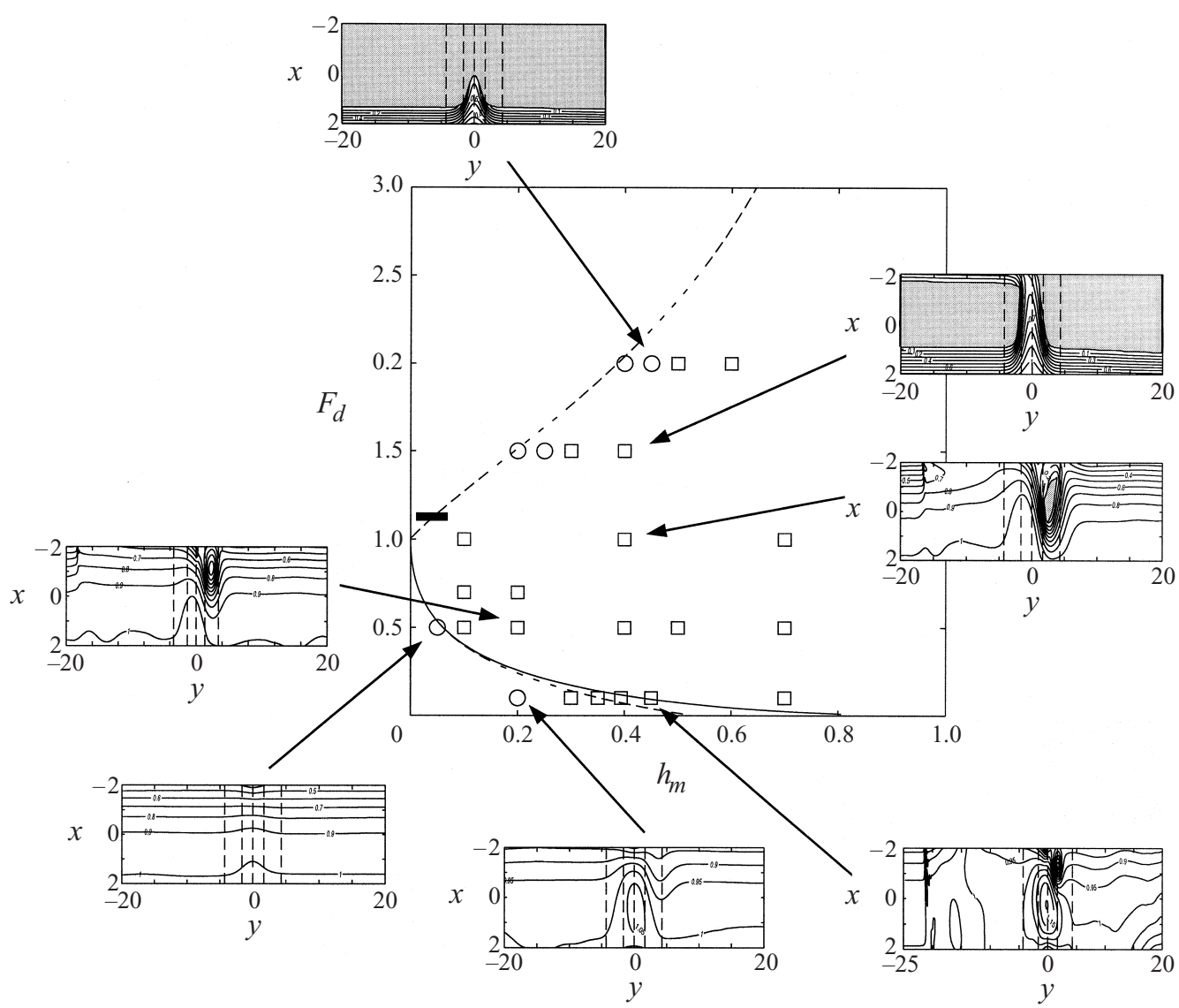

FIGURE 8. Same as figure 6 except $w=4$.

nonlinear example the Kelvin wave does affect the front position, albeit weakly. By $t=20$ the Kelvin wave has propagated out of the domain and the frontal wave (near $y=17$ ) has steepened, nearly to the point where the stream width $w_{e}$ is discontinuous. The result is a transient that looks very much like a family of shock waves discussed by Nof (1984) in connection with a zero-potential-vorticity, separated coastal flow. The last panel at $t=80$ shows the (nearly) symmetric final steady state.

As noted above, upstream influence for the case $w=0.5$ generally occurs where predicted $\left(h_{m}>h_{c}\right)$. When the initial flow is attached, the disturbance that alters the upstream state takes the form of a 'Kelvin-wave' bore. Figure 10 shows an example of this process for $F_{d}=0.5, h_{m}=0.2$. At $t=10$ both upstream- and downstream-propagating Kelvin waves are evident on each side of the topography. The characteristic trapping of the Kelvin waves to the sidewalls is weakly apparent in this narrow channel. By $t=30$ the downstream wave has left the domain, the upstream wave has steepened into a bore, and a hydraulic jump has formed on the downstream side of the obstacle. The jump remains over the topography in the final steady flow $t=50$. The bottom panel of figure 10 shows $F_{d}$ from (2.16) calculated from the numerical solution at $t=30$. The flow makes a transition from subcritical to supercritical over the sill and returns to subcritical across the downstream jump. Also note how $F_{d}$ decreases across the upstream bore. Generally speaking, the solution is 


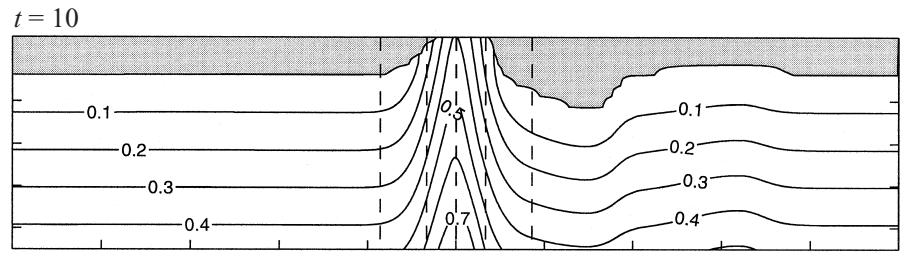

$t=20$
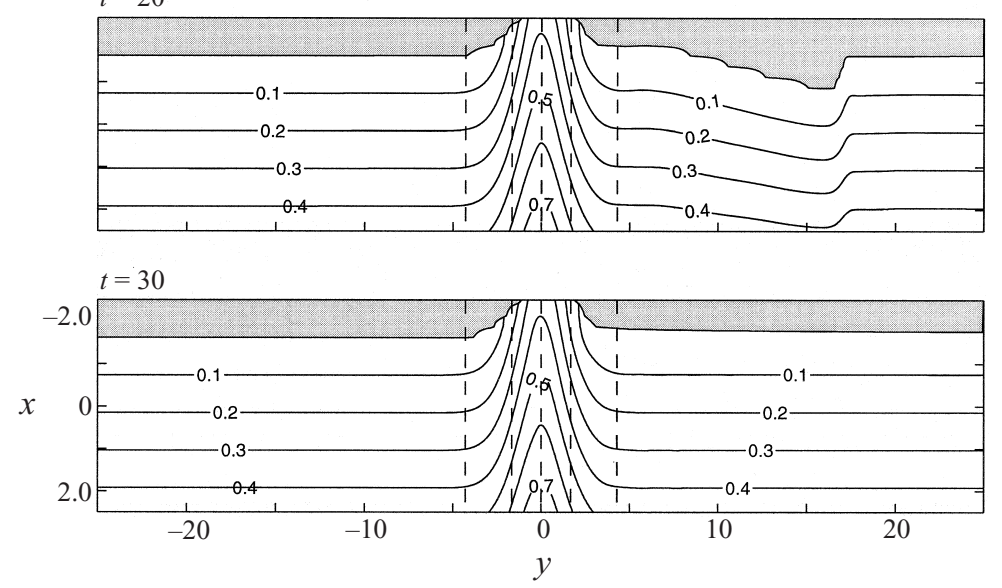

FIGURE 9. Numerical results for $F_{d}=2.5, h_{m}=0.3$ and $w=0.5$. The panels show contours of the free surface height $(d(x, y, t)+h(y))$ at the times indicated. The shaded regions indicate those portions of the channel which are 'dry' (defined by $d<0.001$ ). The dashed lines are the $1,0.5$ and 0.001 times $h_{m}$ contours of the bottom topography.

similar to the non-rotating case. The most apparent sign of rotation is the deflection towards the right-hand wall of the supercritical flow in the lee of the obstacle.

As in the non-rotating case, low values of the initial $F_{d}$ favour stationary hydraulic jumps whereas higher values tend to cause the jumps to move downstream. The latter is illustrated by the inset in figure 6 for for $F_{d}=1$ and $h_{m}=0.2$, where the former hydraulic jump is shown as a discontinuity moving away from the topography in the downstream direction. With no rotation the boundary in the $\left(h_{m}, F_{d}\right)$-plane separating regimes with and without jumps can be constructed analytically using shock joining theory. This boundary is given by the curve $\mathrm{AD}$ in figure 1. A similar calculation is much more difficult in the rotating case due to the unavailability of a satisfactory shock joining theory (Pratt 1983; Nof 1986).

There are also some instances where the supercritical flow downstream of the sill separates from the left-hand wall, a behaviour that has important ramifications for downstream disturbances. As an example, consider the evolution for $F_{d}=1.5$ and $h_{m}=0.5$, as shown in figure 11. At $t=10$ the downstream-propagating Kelvin wave and upstream-propagating bore are evident. The flow approaching the sill is accelerated and veers toward the right-hand wall downstream of the crest, leaving a small patch of dry channel near the left-hand wall. The transition back to attached flow near $y=7$ occurs as an abrupt expansion (located near $y=16$ at $t=30$ ). This transition is swept down the channel, enlarging the dry region $(t=30)$ and ultimately leaving behind a detached supercritical flow in the lee of the topography $(t=50)$. The characteristic speed $c$ has been calculated from (2.15) at points slightly upstream of and slightly downstream of the abrupt transition. On the upstream side, where the 

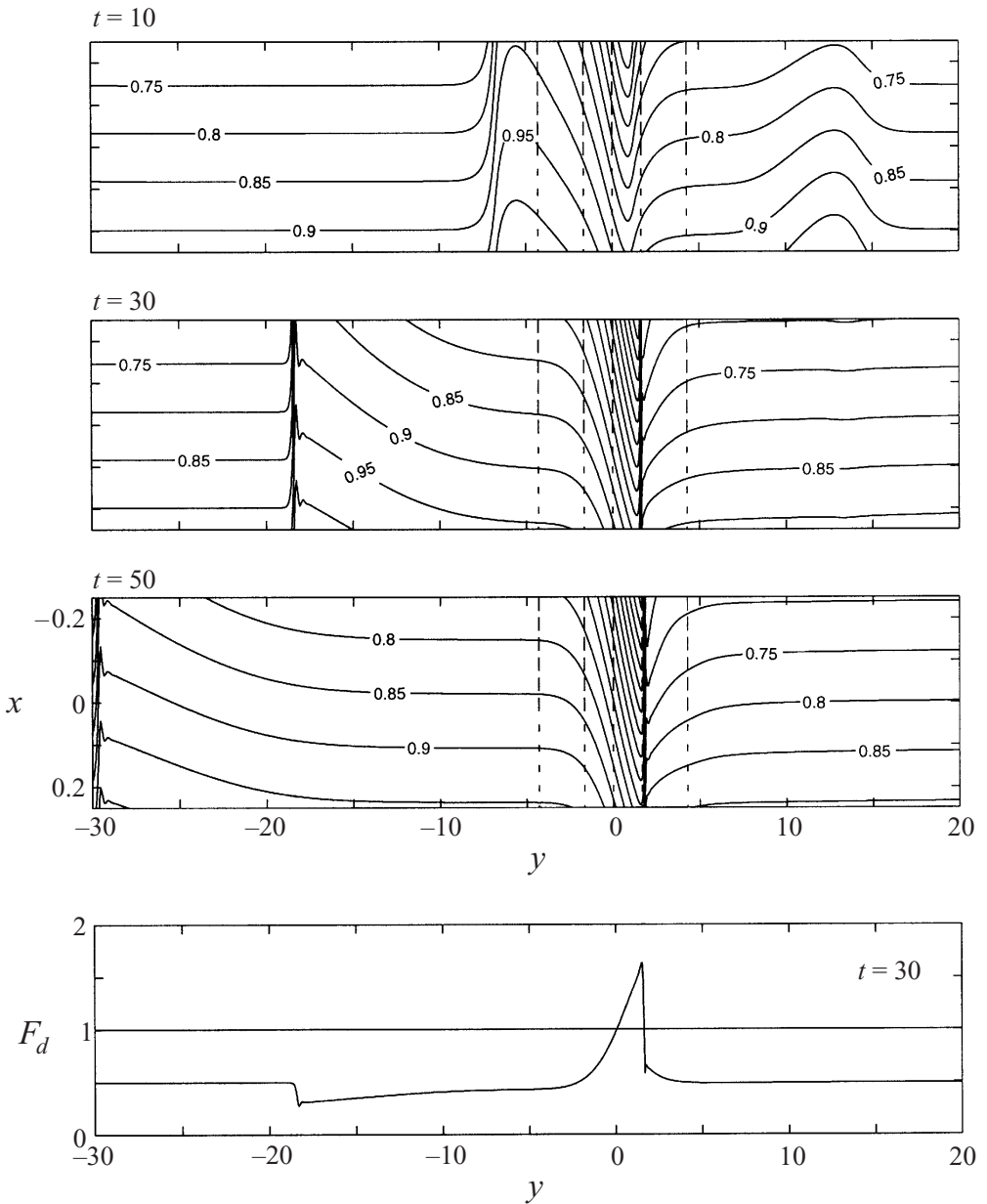

FIGURE 10. Same as figure 9 , except $F_{d}=0.5, h_{m}=0.2$. The bottom panel shows $F_{d}$ as a function of $y$ at $t=30$.

flow is separated and frontal wave dynamics apply, $c$ is positive and greater than on the downstream side, where the flow is attached and Kelvin wave dynamics apply. Thus, linear disturbances generated just upstream of the transition overtake those generated just downstream, indicating that the transition is indeed a shock.

Flow separation in the lee of the obstacle is also observed for subcritical initial conditions and large values of $h_{m}$. In cases where hydraulic jumps occur, the usual abrupt change in depth is replaced by an abrupt change in the width of the stream. That is, the jump is much like the transition in figure 11 , but with the feature stationary in the lee of the topography. An example is shown in figure 12(a) (for $\left.F_{d}=0.5, h_{m}=0.8\right)$ where a small shaded area indicating dry bottom appears in $1<y<2.3$. The separated region terminates in a sudden expansion and resulting reattachment of the flow, downstream of which is a zone of cyclonic recirculation. Figure $12(b)$ shows a plot of $F_{d}$ from (2.16) for this particular example. Also shown is the layer depth on the left-hand wall, $d(-w / 2, y)$. The transition from detached $(d(-w / 2, y)=0)$ to attached flow near $y=2.3$ coincides with the drop of $F_{d}$ from supercritical to subcritical. This 'transverse' jump and trailing recirculation was also 

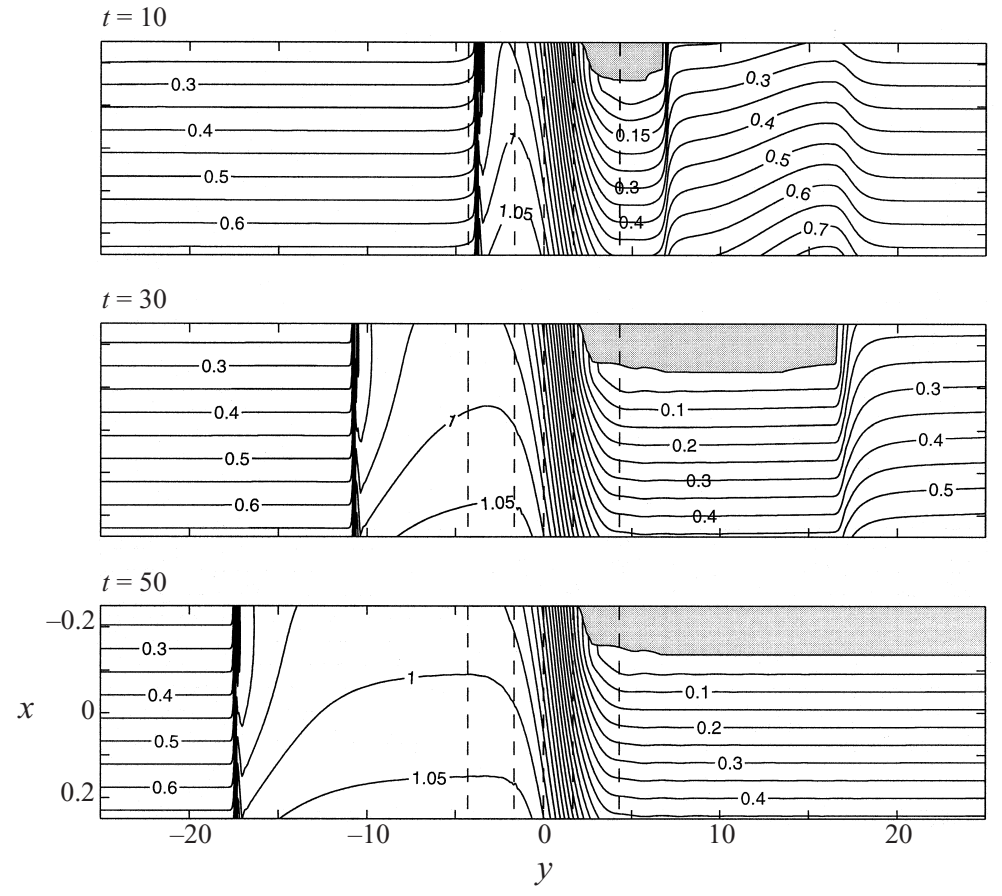

FiguRE 11. Same as figure 9, except $F_{d}=1.5, h_{m}=0.5$.

observed in laboratory experiments by Pratt (1987). In both the stationary and moving version (i.e. the 'transverse' bore) one of the end states is always attached the left-hand wall. The dynamics and temporal development of transverse jumps and bores are discussed in greater detail below.

When the initial flow is separated, upstream influence occurs in an unexpected manner. The leading portion of the upstream-moving disturbance is a rarefying intrusion attached to the left-hand wall as illustrated in the inset in figure 6 for $F_{d}=2.5$ and $h_{m}=0.5$. In this example the intrusion is followed by a surge which leaves behind attached flow upstream of the sill. The surge results in a rapid increase in depth; however, the front is smooth and behaves like a rarefaction rather than a shock. We will return to this interesting situation below.

The last few examples show that rotation can lead to remarkable effects even when $w$ is moderately small. These effects occur where high velocities are present, either due to supercritical initial conditions or because high velocities are induced in the lee of large obstacles. The high velocities lead to strong tilts in the free surface, sometimes resulting in separation of the flow. The Rossby radius of deformation based on the local depth is tiny near the edge of separated current and it is no surprise that inherently rotational features such as the transverse hydraulic jump arise under these conditions.

A final remark about the case $w=0.5$ is that we have not been able to verify the subcritical flow which briefly detaches over the sill (predicted in region DBC in figure 4). Nor have we been able to do so for other values of $w$. More significantly, the hydraulically controlled flows to the immediate right of $\mathrm{BC}$ have attached flow at the sill, despite our prediction to the contrary. This subject is revisited in $\S 5$. 


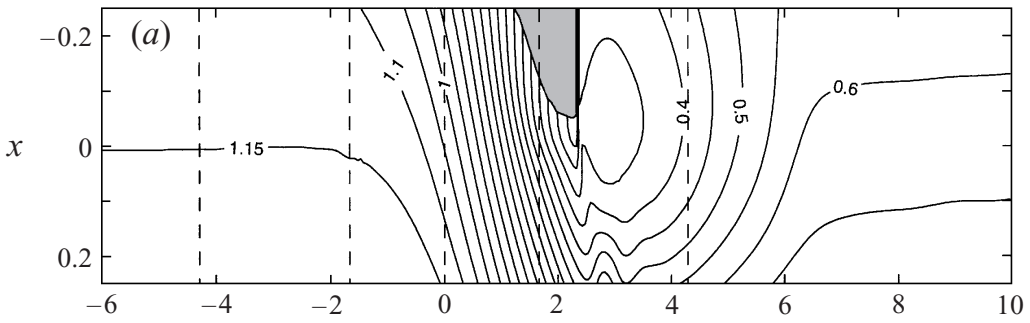

$y$

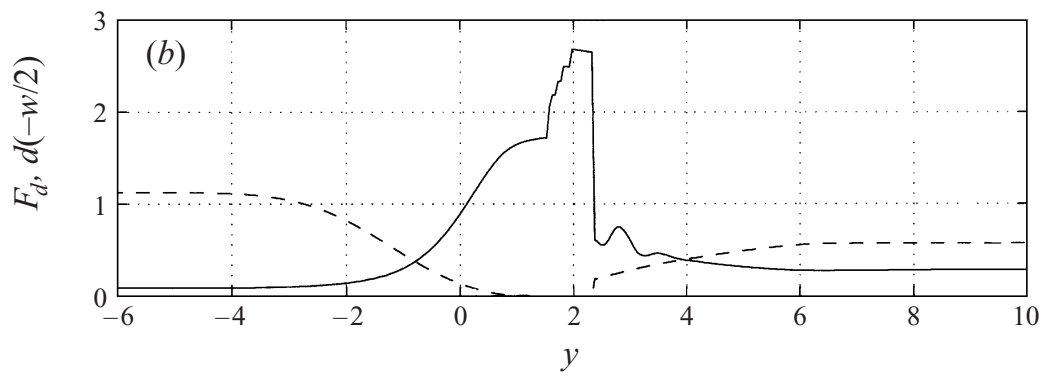

Figure 12. (a) Surface elevation contours for the steady flow that arises in the case $F_{d}=0.5$, $h_{m}=0.8$ and $w=0.5$. A transverse hydraulic jump lies at $y \approx 2.3$. (b) Plot of $F_{d}$ (solid line) and $d(-w / 2, y)$ (dashed line) for the flow in $(a)$. The transition from supercritical to subcritical flow near $y=2.3$ coincides with the lateral expansion and reattachment of the flow to the left-hand wall.

\subsubsection{Cases $w=2$ and $w=4$}

The regime diagrams for the cases $w=2$ and $w=4$ are shown in figures 7 and 8 . Separation of the initial flow now occurs for nearly all $F_{d}>1$, as indicated by dashing of the critical obstacle height curve. The region analogous to $A^{\prime} \mathrm{HG}$ of figure 4 is indistinguishably thin in each of figures 7 and 8 , and therefore the upper boundary of this region is indicated by a horizontal bar. This bar marks that value of $F_{d}$ above which the predicted critical sill flow is separated.

An immediate consequence of the greater $w$ is that it shrinks the range of $F_{d}$ and $h_{m}$ over which the flow remains attached at all sections. Upstream influence in cases of complete attachment still occurs through the propagation of a 'Kelvin wave' bore. An example for $F_{d}=0.5, h_{m}=0.2$ and $w=2$ is shown in figure 13. In this case both an upstream bore and a downstream hydraulic jump are generated. In contrast to the case $w=0.5$ (cf. figure 10) neither the bore nor the jump extend across the channel, but rather are strongly trapped to the left-hand wall. The downstream jump has a lateral scale of only about 0.25 , or half of a deformation radius. These flows resemble the solutions computed by Pratt (1983). Downstream of the jump a region of cyclonic recirculation is generated. This region appears to expand in the downstream direction indefinitely.

The amplitude of the 'Kelvin wave' jump remains small as long as $h_{m}$ is not greatly exceeded. In fact cases such as $\left(F_{d}=0.5, h_{m}=0.5\right)$ and $\left(F_{d}=1, h_{m}=0.1\right)$ of figure 7 show very little evidence of jumps, at least in the free-surface elevation. These flows also contain cyclonic recirculations in the lee of the obstacle. Some of the details of the transition from supercritical to subcritical flow in cases such as these are discussed in the next section.

When $h_{m}$ is increased well beyond the critical value the lee flow detaches, then reattaches over the topography to form a transverse jump as discussed above. An 

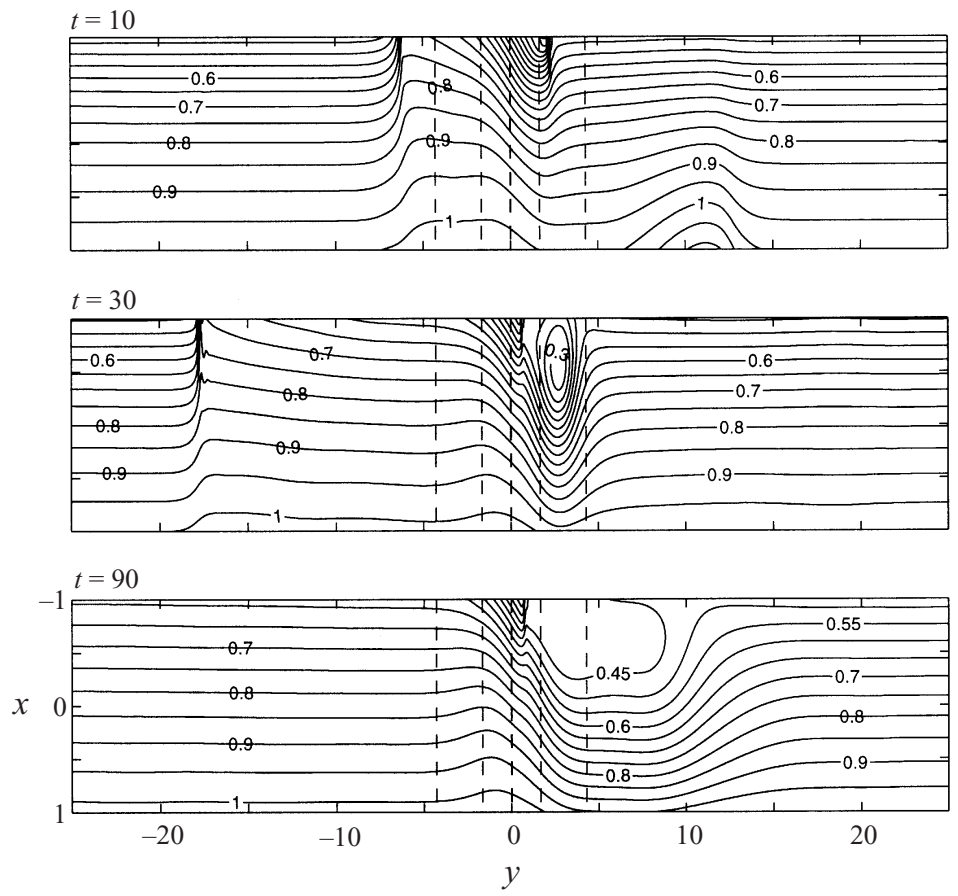

FIGURE 13. Same as figure 9, but now for the case $w=2$, and $F_{d}=0.5, h_{m}=0.2$.

example of the development of this flow is shown in figure 14 for $F_{d}=1, h_{m}=0.5$ and $w=2$. At $t=10$ a large dry region has formed on the downstream side of the topography. This zone splits into a small wedge of dry channel and a large, intense cyclonic eddy with a dry centre $(t=30)$. The outcropping in the eddy fills in by $t=50$ to form an expanding zone of cyclonic recirculation and the transverse jump remains fixed to the topography.

For supercritical initial conditions the flow is separated for all Froude numbers save those close to unity. Figure 15 shows the evolving flow for a separated supercritical case with $F_{d}=1.5, h_{m}=0.4$ and $w=2$. The predicted critical flow at the sill is attached in this case. Upstream influence occurs in the form of bifurcation of the initial current over the topography. A portion of the incident flow is diverted back towards negative $y$, forming a separated, rarefying intrusion along the left-hand wall, while the rest continues over the topography. The original current is narrow enough that the upstream intrusion does not contact the original current in the neighbourhood immediately upstream of the topography. The final steady state upstream of the topography consists of two opposite, separated currents. Remarkably, there is no upstream influence in the original current. However, the net flux towards the sill is reduced by the diversion of fluid into the left-wall intrusion. This case contrasts with the example discussed earlier for $w=0.5$ where the upstream intrusion was followed by a surge occupying the full width of the channel. Which situation occurs depends upon the width of the initial current, $w_{e}$ compared to $w$. We have not fully explored the change in behaviour, but the full-width surge occurs for $w_{e} / w=O(1)$ while the detached intrusion occurs for small values of $w_{e} / w$. Over the upstream face of the obstacle the flow fills the channel and becomes subcritical. This subcritical flow becomes critical at the sill and supercritical (and eventually detached) downstream. 

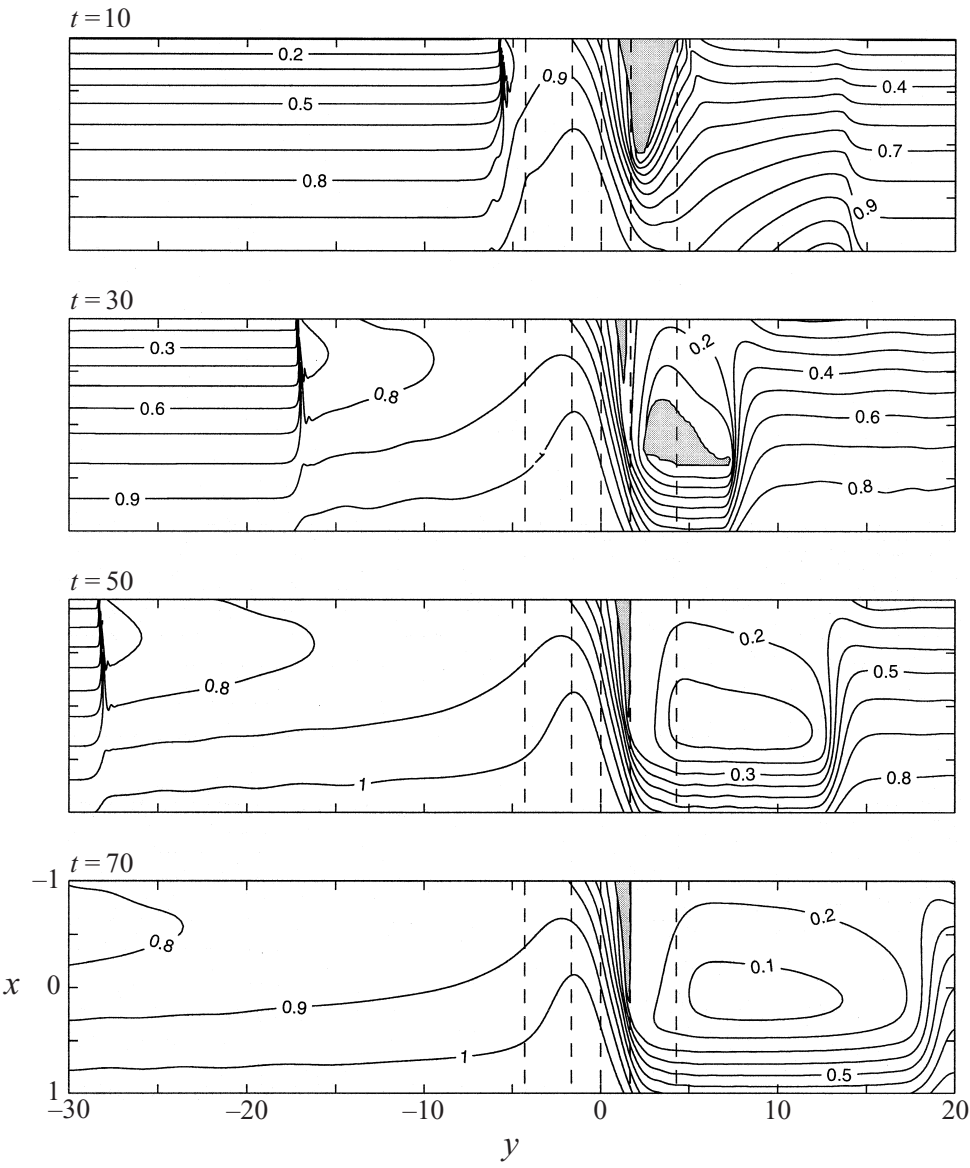

FIGURE 14. Same as figure 13, except $F_{d}=1, h_{m}=0.5$.

A frontal wave is observed further downstream $(y=9$ at $t=20)$. Note the ejection of a blob of fluid (near $y=12$ at $t=40$ ) as a result of steepening of this wave.

The branching of the stream described above (near $y=-2.5$ at $t=80$ in figure 15) is essentially a dissipationless shock. The upstream end state consists of two supercritical streams, both separated, while the downstream end state consists of an attached subcritical stream. Given one end state, it should be possible to predict the other by invoking conservation of mass, momentum and potential vorticity across the branching section. This exercise has not been pursued.

We have also performed calculations based on large enough values $F_{d}$ so that the predicted critical sill flow is separated. Such values lie above the horizontal bar in figures 7 and 8. Significantly, these settings also result in left-wall intrusions of the type just discussed and in attached flow at the sill. An example is shown in the figure 8 inset for $F_{d}=1.5, h_{m}=0.4$ and $w=4$. In no cases have we observed sill flows which are critical and separated at the sill.

In the case of the widest channel considered $w=4$, the flow responds much as in the $w=2$ case. However, one qualitative difference, evident for flows with $F_{d}<0.5$, is the appearance of an anticyclonic recirculation cell over the sill. This feature arose regardless of the occurrence of upstream influence as illustrated by the two insets in figure 8 for $F_{d}=0.1$ and $h_{m}=0.2$ and 0.45 . In both cases the velocity 

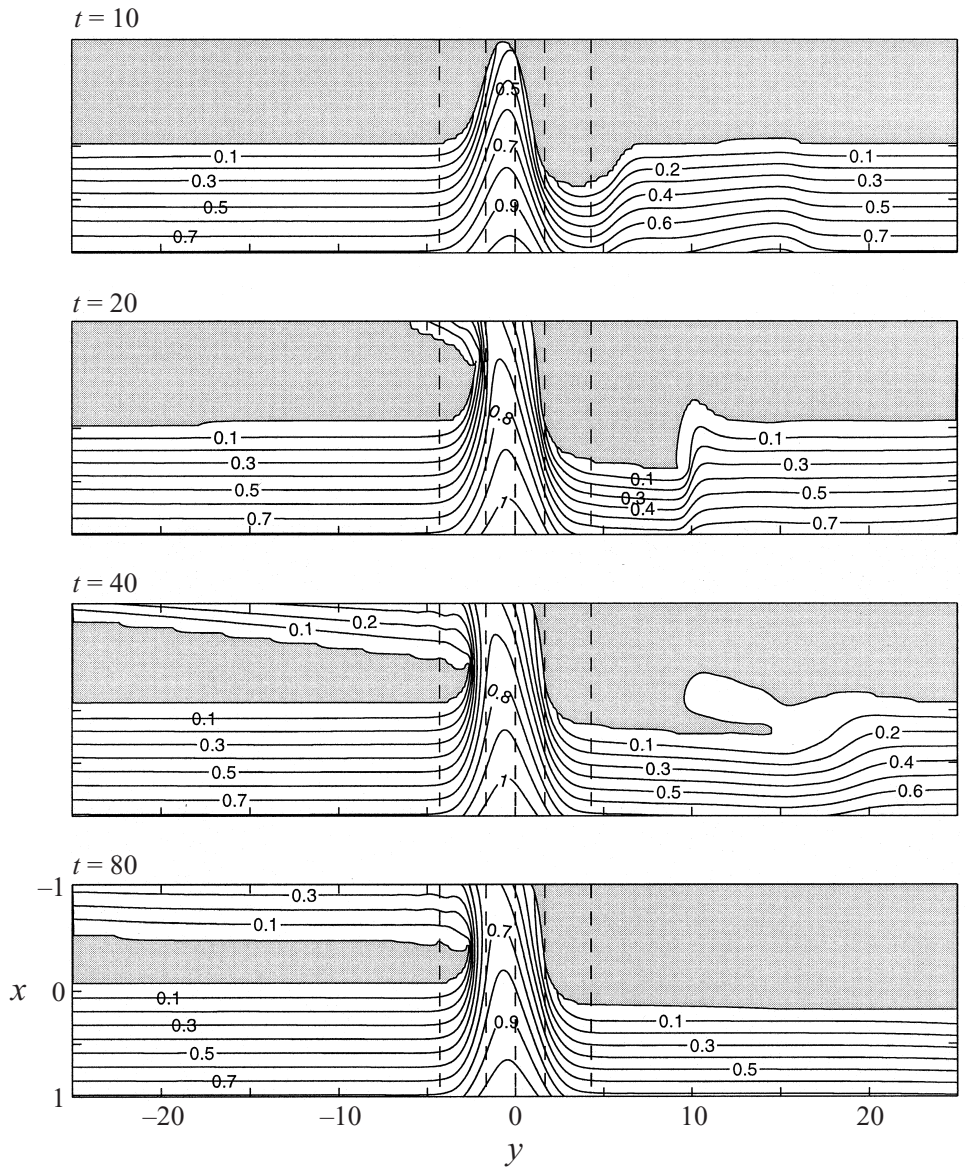

Figure 15. Same as figure 13 , except $F_{d}=1.5, h_{m}=0.4$.

on the right-hand wall at the sill crest $v(w / 2,0)<0$. The recirculation cell occupies about three-quarters of the channel width, forcing the fluid which crosses the sill and continues downstream to do so in a narrow band adjacent to the left-hand wall. The along-channel extent of the recirculation is comparable to the length of the topography.

\section{Discussion}

\subsection{Failure of hydraulic control in separated sill flows}

Both of the poineering models of steady, hydraulically driven flow in rotating channels (Whitehead et al. 1974 and Gill 1977) describe solutions which are hydraulically critical and separated at the controlling sill or narrows. Examples can be found in Gill's (1977) figures 6 and 7, and 9(d). Surprisingly, laboratory experiments by Shen (1981) and Pratt (1987) failed to produce such flows, even though attempts were made to do so. Whitehead et al. (1974) claimed to have achieved separated and hydraulically controlled flow in the laboratory, but their 'sill' was actually a finite-length segment of rectangular channel over which the bottom elevation and width is constant. Although the flow is separated in the downstream portion of this segment, it is not at the 
upstream portion. The exact section of critical flow and its state of separation are unknown.

It is significant that our numerical simulations have failed to produce hydraulically controlled flows that are separated at the sill section. Such states might have been expected as a result of runs such as $F_{d}=1.5$ and $h_{m}=0.4$ of figure 8 , in which the initial flow is separated and the predicted sill flow is also separated. However, the obstacle causes deflection of the approaching flow to the left-hand wall resulting in the formation of an intrusion as shown in figure 15 . Over the sill itself, the flow becomes attached. Other cases where the sill flow is predicted to be separated and critical correspond to very subcritical, attached initial flows and large $h_{m}$ (as in the region just to the right of curve $\mathrm{BC}$ in figure 4). Although the numerical experiments confirm that the flow is controlled, it remains attached at the sill.

These findings seem to suggest the presence of an instability that acts when a separated flow is critical or subcritical. However, Paldor (1983) has shown that separated currents of the type under discussion are stable, at least in the limit of zero potential vorticity, provided that the fluid depth along the right-hand wall remains non-zero. So there does not yet appear to be a clear connection between inviscid instability and the lack of separated critical flow in the numerical experiments.

A second possible cause is the apparent inability of bores in separated flows to propagate upstream. Nof (1984) has discussed this problem in connection with a model of shock waves in a separated, zero-potential-vorticity flow. Although there may be some dynamical inconsistencies in his solutions due to an assumption of potential vorticity conservation through the shock, his results are very suggestive. For example, it can be shown from the zero-potentail-vorticity depth and velocity profiles that the total volume transport across any section increases as the width of the stream increases. This property holds as long as the velocity remains positive $v>0$ at all $x$. Therefore a bore having a narrow upstream, and wide downstream, end state will have larger volume transport across the downstream end state. If the bore translates steadily, conservation of mass then requires that it propagate in the $+y$-direction. In the figure 15 solution at $t=10$, the separated initial flow collides with the obstacle, resulting in a widening of the current. However, an upstream disturbance is not generated until contact with the left-hand wall is made-perhaps due to the reasons just mentioned. $\dagger$

\subsection{The distribution of dissipation in an attached jump}

We have observed that a hydraulic jump forming under attached conditions resembles a breaking Kelvin wave. The jump amplitude is largest at the left-hand wall and decays away from that wall over a distance $\approx L_{d}$. It would appear that such a jump would lead to dissipation (and overturning, if such was explicit in the model) primarily along the left-hand wall. The actual distribution of dissipation can be more subtle and we now show this using an example of a highly localized jump.

An enlarged picture of the surface elevation contours for the case $\left(F_{d}=0.5, h_{m}=0.5\right.$ and $w=2$ ) is shown in figure $16(a)$. The free surface is smooth, except for a weak hydraulic jump along the left-hand wall near $y=1$. Very little transport (about $\frac{1}{8}$ of the total) actually passes through the jump. The usual recirculation exists downstream. The jump appears more clearly in figures 16(b) and 16(c), where contours of energy

$\dagger$ If one allows negative $v$ to form in the downstream end state, then that state need no longer carry greater transport than the upstream end state. In this case, a separated and upstream-propagating bore would not violate mass conservation. Whether such a disturbance is dynamically possible is unknown. 
(a)

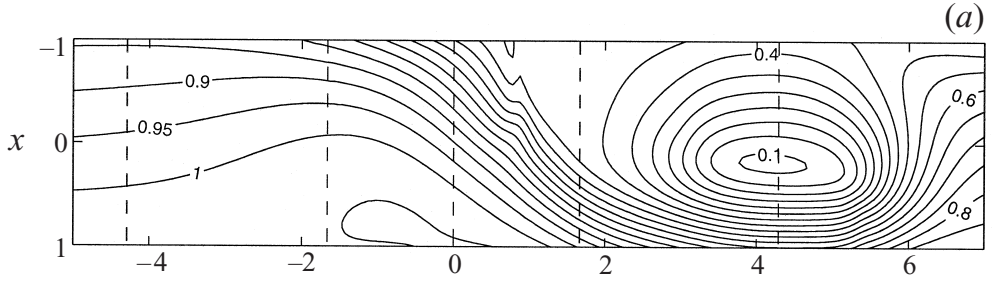

(b)

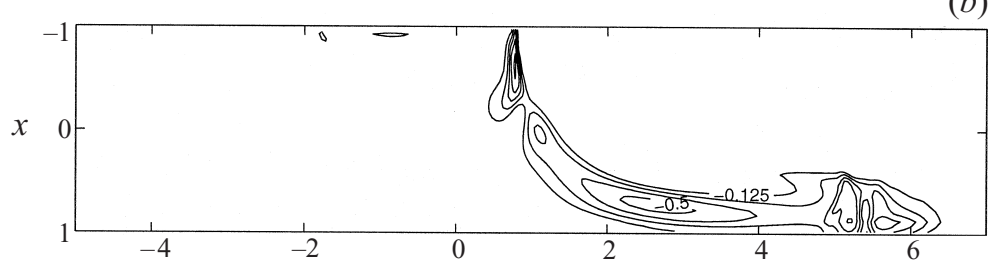

(c)

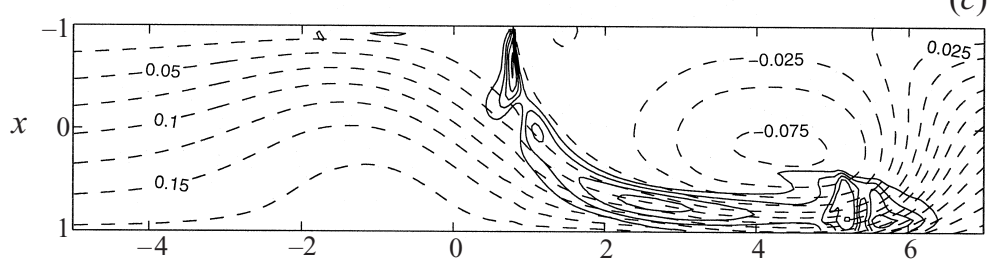

(d)

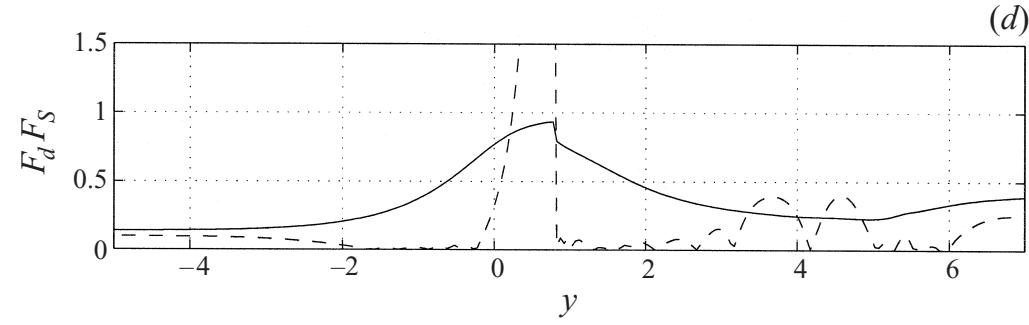

FiguRE 16. Details of the super- to subcritical transitions for the inset in figure 7 ( $w=2$ at $F_{d}=0.5$ and $h_{m}=0.5$. (a) Contours of the free-surface elevation $d+h$. (b) Contours of the dissipation $\mu \boldsymbol{u} \cdot(\nabla \cdot(d \nabla \boldsymbol{u}))$. The contour interval is $1.25 \times 10^{-2}$. (c) Contours of the transport streamfunction (dashed lines) overlaid with the dissipation contours from above. (d) The Froude number $F_{d}$ based on equation (2.16) (solid line) and $F_{S}$ based on equation (5.2) (dashed line). The definition of $F_{S}$ is invalid downstream of the jump (at $y \approx 1$ ) due to velocity reversals.

dissipation $(\mu \boldsymbol{u} \cdot(\nabla \cdot(d \nabla \boldsymbol{u})))$ and dissipation overlaid by streamlines are plotted. Two regions of high dissipation can be distinguished, one coincident with the jump and the second further downstream and closer to the right-hand wall. This second region is one of high velocity and shear $(\partial v / \partial x)$, formed where the throughflow is squeezed along the right-hand wall by the recirculation. The maximum dissipation values in the latter region are comparable to those in the jump region. In addition the region of high dissipation due to shear is much larger than the jump region. Clearly, all fluid experiences dissipation as the jump and recirculation is passed. 


\subsection{Breakdown of semigeostrophic theory}

Large channel widths permit the formation of larger cross-channel velocities, leading the departures from semigeostrophic behaviour. One way to document such departures is through the semigeostrophic Froude number. Figure 16(d) contains a longitudinal plot (solid line) of the constant-potential-vorticity, semigeostrophic Froude (2.16) for the flow shown frames $(a-c)$. Suprisingly $F_{d}$ never exceeds unity, reaching a maximum value $\approx 0.95$ just upstream of the hydraulic jump. The value at the sill is considerably lower. The fact that this example clearly exhibits upstream influence (and that something like a hydraulic jump exists) suggests $F_{d}$ should equal 1 at the sill and exceed 1 immediately downstream. Apparently (2.16) is no longer a reliable definition of the Froude number. As shown in figure 16(c), significant cross-channel velocities exist near and slightly downstream of the sill, suggesting that the failure of (2.16) may be due to a loss of semigeostrophy. This failure could also be due to potential-vorticity non-uniformity that might have developed in the flow field.

To test the second hypothesis, we have formulated a more general Froude number that is valid for non-uniform potential-vorticity flow but requires semigeostrophic conditions. First consider the generalized critical condition for semigeostrophic flow derived by Stern (1974):

$$
\int_{-w / 2}^{w / 2}\left(v^{2} d\right)^{-1}\left(1-\frac{v^{2}}{d}\right) \mathrm{d} x=0 .
$$

The derivation of (5.1) is valid only when $d$ remains positive and $v$ remains single signed in $-w / 2 \geqslant y \geqslant w / 2$. From this definition we can formulate a generalized Froude number $F_{s}$, defined by

$$
F_{S}^{2}=\int_{-w / 2}^{w / 2} d^{-2} \mathrm{~d} x / \int_{-w / 2}^{w / 2}\left(v^{2} d\right)^{-1} \mathrm{~d} x,
$$

noting that $F_{S}=1$ when the flow is hydraulically critical and $F_{S} \rightarrow 0$ as $v \rightarrow 0$. Figure $16(d)$ contains a plot (the dashed line) of $F_{S}(y)$ along with $F_{d}$ (solid line). Although $F_{S}$ does exceed unity on the downstream face of the obstacle, its value $(\approx 0.4)$ at the sill is even lower than the value of $F_{d}$ there. (The definition of $F_{S}(y)$ downstream of the transverse jump breaks down due to flow reversals.) This indicates that the breakdown in our measure of the Froude number is due to the failure of the semigeostrophic approximation. This conclusion is supported by the behaviour of the Froude number in a flow regime with the same obstacle height but a narrower channel $\left(w=0.5\right.$, bottom panel of figure 10). Here $F_{d}$ reaches and exceeds 1 in the expected places.

In addition to the failure of (2.16) to measure the true Froude number of the flow, there are other indications of breakdown of the semigeostrophic approximation. The value of the critical obstacle height $h_{c}$ predicted by semigeostrophic theory agrees well with the observed values for our narrowest channel $(w=0.5)$. As $w$ increases the agreement grows worse: the predicted $h_{c}$ overestimates the actual $h_{c}$ for subcritical initial flows and underestimates it for supercritical initial flows. This trend is probably a result of the fact that large cross-channel velocities are allowed to develop once the channel width exceeds a deformation radius. Despite these differences the general shape of the curve of $h_{c}$ as a function of $F_{d}$ remains as predicted.

More striking breakdowns in the semigeostrophic approximation occur within individual features. Perhaps the most dramatic is the grounding or separation of the flow $(d \rightarrow 0)$ in the interior of the stream, as occurs at the bifurcation of the upstream 
flow $(t=20,40$, and 80 in figure 15 near $y=-2.5$ ), at the detaching eddy (near $y=14$ and $t=40$ of the same figure), and at the cyclonic vortex with the 'dry' interior $(t=30$ in figure 14 near $y=5.0)$. Such behaviour is clearly in violation of Gill's (1977) theorem proscribing the vanishing of $d$ at a point where $\partial^{2} d / \partial x^{2}>0$ in any semigeostrophic flow (see $\S 2$ ). Not surprisingly, semigeostrophic theory also fails in the vicinity of jumps, bores and other transients exhibiting rapid transitions in the $y$-direction. It is not necessary that $w$ be large for such violations to occur, as evidenced by the presence of transverse jumps and bores when $w=0.5$ (figure 6).

\subsection{Conservation of properties across transverse jumps}

A major deficiency in current rotating hydraulic theory is the lack of a satisfactory shock-joining theory. Consider two sections A and B located slightly upstream and downstream of a stationary jump and suppose that the flow at $\mathrm{A}$ is known. Then the object of shock-joining theory is to predict the flow at B. One approach is to assume that potential vorticity is conserved across the jump, so that the potential vorticity distribution $q_{B}(\psi)$ at $B$ is known. If the flow there is semigeostrophic, then the depth obeys the equation

$$
\frac{\partial^{2} d}{\partial x^{2}}-q_{B}(\psi) d=-1,
$$

which is just the generalization of (2.7) for arbitrary potential vorticity. In principle, (5.3) could be solved at $B$ yielding a depth profile with two unknown integration constants. Equating the cross-sectionally integrated volume flux $v d$ and flow force $v^{2} d+\frac{1}{2} d^{2}$ at $\mathrm{A}$ and $\mathrm{B}$ would then yield the values of these constants.

Attempts by Pratt (1983) and Nof (1986) to apply this method to rotating shocks in attached flows have met with two difficulties. First, the discontinuity in depth or width is generally bordered by a non-semigeostrophic region extending several deformation radii upstream and/or downstream. (Further examples arise in recent models of Fedorov \& Melville 1996 and Helfrich et al. 1999.) The bounding sections A and B must be situated outside these regions, making the length of the shock several deformation radii. Over this distance, continuous momentum sources such as bottom friction or Coriolis acceleration might be large enough to wreck conservation of flow force between A and B. A second difficulty is that potential vorticity is generally not conserved across the depth discontinuity. This property that can be deduced from the relation between the Bernoulli function and potential vorticity

$$
\frac{\mathrm{d} B}{\mathrm{~d} \psi}=q .
$$

Jumps such as the one shown in figure 16(b) clearly experience different energy dissipation levels along different streamlines, and this implies that $q$ must change along those streamlines. Therefore, $q_{B}(\psi)$ is generally unknown. Further examples are discussed by Pratt (1983), Nof (1986), Schär \& Smith (1993), and Helfrich et al. (1999).

It is not clear that these difficulties apply to a transverse hydraulic jump. For one thing, transverse jumps experience no sudden depth change and therefore contain no obvious source of energy dissipation. However, a closer examination reveals that neither potential vorticity nor flow force is generally conserved. Consider the jump located near $y=1.6$ in figure $17(a)$, for which the ageostrophic region extends to $y \approx 7$. We therefore situate section A at $y=1.6$ and section B at $y=7$, as shown in the figure. This interval contains the upstream portion of cyclonic recirculation, an 

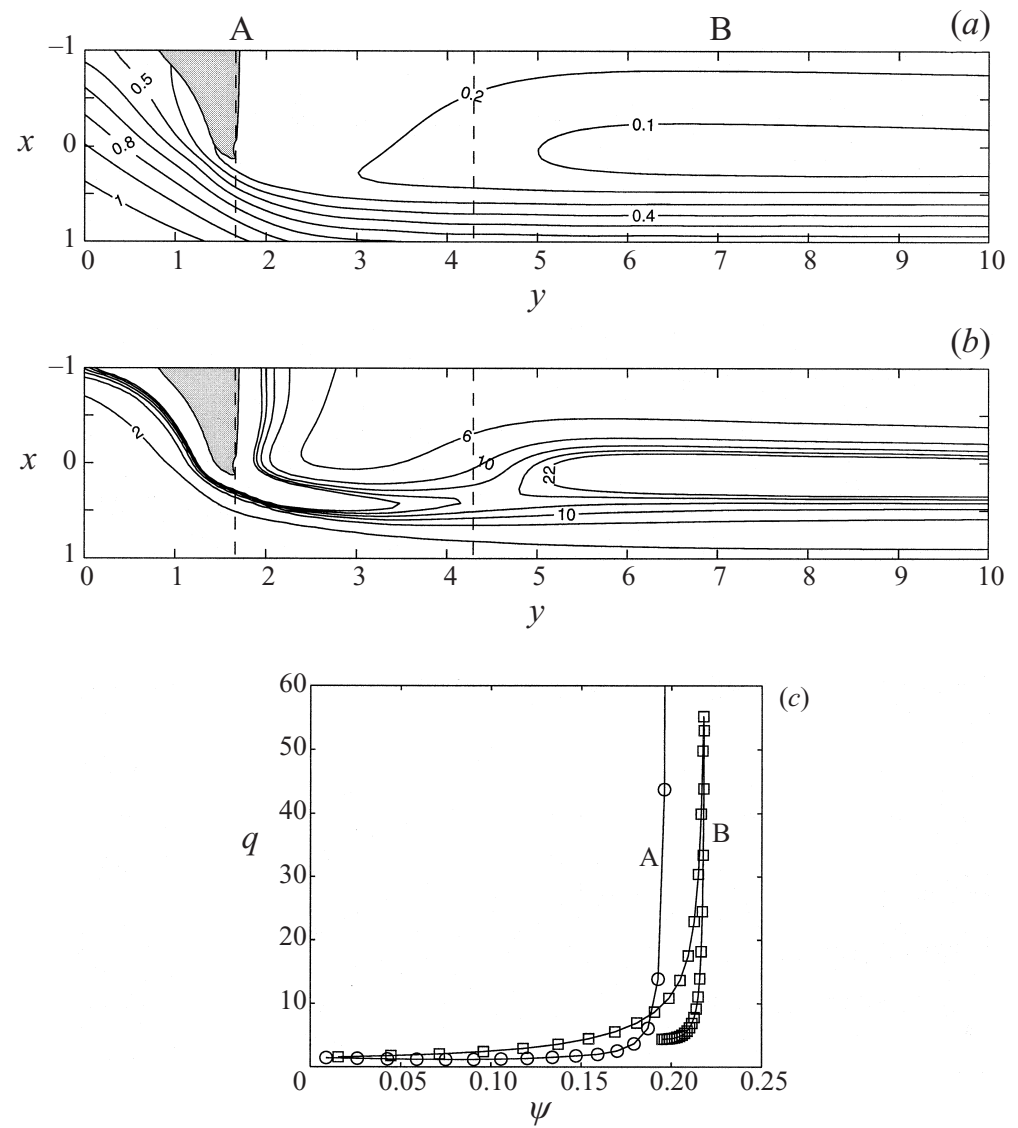

FIgURE 17. (a) Contours of surface elevation in the vicinity of the transverse hydraulic jump for the run in figure $14\left(w=2, F_{d}=1\right.$, and $\left.h_{m}=0.5\right)$ at $t=70$. The sill of the obstacle lies at $y=0$. (b) Potential vorticity distribution for the flow in $(a)$. (c) $q(\psi)$ distributions at sections A and B as marked in $(a)$.

area clearly non-semigeostrophic $[v / u \sim O(1)]$. The $y$-momentum budget, formulated by integrating (4.2) over the area between sections $\mathrm{A}$ and $\mathrm{B}$, may be written as

$$
[M]_{A}^{B}=I_{C}+I_{T}+[D]_{A}^{B},
$$

where

$$
\begin{aligned}
M & =\int_{-w / 2}^{w / 2}\left(v^{2} d+\frac{1}{2} d^{2}\right) \mathrm{d} x, \quad I_{C}=-\iint_{R}(u d) \mathrm{d} \sigma, \quad I_{T}=-\iint_{R} d \frac{\partial h}{\partial y} \mathrm{~d} \sigma, \\
D & =v \int_{-w / 2}^{w / 2} d \frac{\partial v}{\partial y} \mathrm{~d} x, \quad \text { and }[()]_{A}^{B}=()_{B}-()_{A} .
\end{aligned}
$$

We find $M_{A}=0.202$ and $M_{B}=0.260$, so that there is a $29 \%$ gain in flow force across the jump. This gain is provided by the topographic form drag $I_{T}=0.107$, which is partially counteracted by the term $I_{C}=0.045$ resulting from the Coriolis acceleration due to the cross-channel flow in the recirculation. The dissipation term $D$ is negligible.

Contours of potential vorticity are shown in figure $17(b)$ and the distributions $q(\psi)$ at sections A and B are shown in figure 17(c). It is clear from both panels that $q(\psi)$ is very different at the two sections. Starting from the left-hand edge $(\psi=0)$ in figure $17(c)$, corresponding to the right-hand wall of the channel, we see that $q(\psi)$ 
(a)

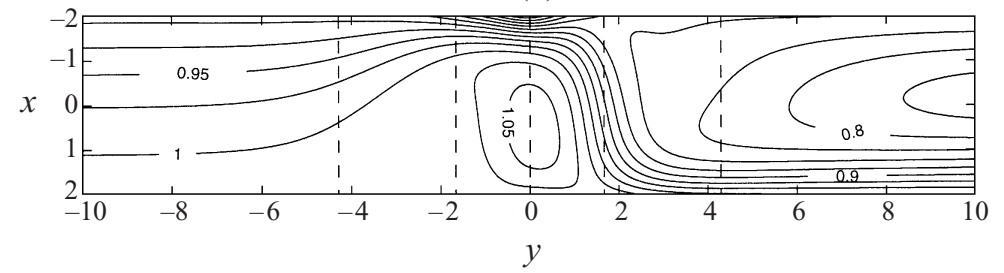

(b)

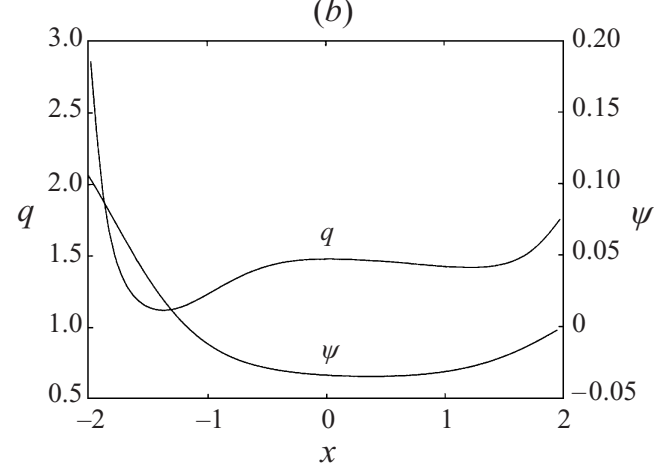

FIGURE 18. (a) Surface height contours for the case $\left(w=4, F_{d}=0.1\right.$, and $\left.h_{c}=0.45\right)$ in which a recirculation exists over the sill. $(b)$ Potential vorticity and streamfunction profiles at the sill $(y=0)$.

at both sections increases gradually with increasing $\psi$ until about $\psi \approx 0.18$, where $q_{A}(\psi)$ suddenly grows off scale. This $\psi$ value marks the free edge of the stream, where $q$ is likely to be sensitive to the dissipation used in the model. Following $q_{B}(\psi)$ to higher $\psi$ values brings one into the recirculating fluid at section $\mathrm{B}$. The maximum value of $q_{B}(\psi)$ at $\psi \approx 0.21$ corresponds to the centre of the recirculation. Following the $q_{B}(\psi)$ curve further leads back to lower values of $\psi$, and lower and more uniform values of $q_{B}$, corresponding to fluid along the left-hand wall. In summary differences in potential vorticity from $\mathrm{A}$ to $\mathrm{B}$ arise due to high dissipation rates near the free edge of the flow at $\mathrm{A}$ and due to the occurrence of $\psi$ values at $\mathrm{B}$, associated with reirculating fluid, that lie outside the $\psi$ range at $\mathrm{A}$.

The discouraging conclusion, then, is that transverse shocks experience the same non-conservation of flow force and potential vorticity that prevent a simple treatment of rotating shocks in attached flows.

\subsection{Upstream recirculations}

Although semigeostrophic theory admits solutions with closed recirculations, the location of the recirculations may be restricted by the assumed potential vorticity distribution. In Gill's (1977) uniform-potential-vorticity model, for example, it can be shown that the flow at any critical section must be unidirectional. Recirculations must therefore occur away from control sections. Borenäs \& Whitehead (1998) did find analytical and laboratory solutions with upstream recirculation, but in all cases the recirculation zone terminates at, or before, the sill crest. The potential vorticity distribution in their laboratory flows were not known. Their analytical solutions had uniform potential vorticity outside the recirculation and either (the same) uniform potential vorticity or stagnant conditions inside. On the other hand, flows with nonuniform potential vorticity may contain any number of zero crossings at a control section and examples of this behaviour have been given by Pratt \& Armi (1987). 
Figure 18(a) shows the recirculation that forms over the obstacle for the case ( $w=4, F_{d}=0.1$, and $h_{c}=0.45$ ). (The flow at this stage is still undergoing a slow temporal evolution, perhaps due to potential vorticity dynamics.) Recirculating fluid clearly exists at the sill section. Inspection of the potential vorticity distribution across the sill confirms that it is non-uniform. Figure 18(b) shows profiles of both $q$ and $\psi$ at the sill, the observer facing downstream. The boundaries of the recirculation correspond to $\psi=0$, which occurs at the right-hand wall $x=2$ and at $x \approx-1$. Within these boundaries $q$ is roughly constant, in agreement with conditions conjectured by Borenäs \& Whitehead (1998). To the left of the recirculation the potential vorticity is much higher.

\subsection{Comparison with the case of lateral uniformity}

Baines \& Leonard (1989, hereafter referred to as BL) have presented solutions describing the adjustment of a uniform stream (constant $v_{0}$ and $d_{0}, u_{0}=0$ ) to a ridge on an infinite $f$-plane. $\dagger$ The topography is uniform in $x$ and therefore the flow remains $x$-independent for all time. There are some important qualitative differences between the $t \rightarrow \infty$ states found by $\mathrm{BL}$ and in the present experiment. The most striking is that the upstream effects of the ridge are confined to a finite distance from the topography. When the ridge height exceeds a critical value, an upstream bore is generated as in the present experiment. However, the pressure gradient due to the surface slope $[\partial(d+h) / \partial y]$ in the bore gives rise to a geostrophic current $(u<0)$ parallel to the ridge. In our channel problem this current would collide with the left-hand wall and generate a Kelvin wave, the influence of which would be felt far upstream. In BL the current continues unimpeded. As the bore travels upstream its amplitude and speed are reduced and eventually the bore becomes stationary. The $t \rightarrow \infty$ solution upstream of the obstacle consists of this arrested bore, followed by a band of transverse flow. These features occur within a deformation radius of the upstream edge of the obstacle; further upstream the flow remains undisturbed.

A second departure from the present problem is in the nature of the critical condition. Although Kelvin waves are not possible in the BL model, there is still a critical condition with respect to short inertia-gravity waves. The authors show that this condition must occur where $u=-\partial h / \partial y$, in contrast with the present criterion $\partial h / \partial y=0$.

\section{Summary}

One of the primary aims of this work has been to establish a set of regime diagrams for a rotating version of Long's experiment with a homogeneous fluid. Although lack of a satisfactory joining theory for rotating shocks has prevented prediction of all of the corresponding regime boundaries, we have been able to find some. These include the curve of critical obstacle height $h_{c}\left(F_{d}, w\right)$ as well as other curves separating different states of flow separation. All boundary predictions are based on semigeostrophic theory for uniform-potential-vorticity flow. The regime diagrams also include insets showing representative examples based on numerical integration of the full, two-dimensional shallow water equations. It is hoped that the results (figures 6, 7, and 8) will be helpful to investigators who wish to learn about rotating hydraulics.

$\dagger$ In order to keep the initial flow uniform and geostrophically balanced, the authors add an externally imposed pressure gradient in the $x$-direction. 
In reviewing figures $6-8$, most of the examples of hydraulically controlled flow can be placed in two broad classes. The first includes flows that remain attached to the left-hand wall at each $y$. The time-dependent adjustment leading to the establishment of a controlled flow in this regime is similar to what takes place in Long's experiments, although the transients and the hydraulic jumps become trapped to sidewalls. One might collectively refer to these examples as the Kelvin-wave regime. This regime generally occurs for small-to-moderate $F_{d}, h_{m}$, and $w$. The second category includes flows that are separated from the left-hand wall over some $y$. Significantly, the sill flow in all such cases remains attached. Further, all upstream disturbances and hydraulic jumps with separated upstream flow have attached downstream end states. Both Kelvin-wave and frontal-wave dynamics are important in these examples, which might collectively be referred to as a 'hybrid' regime. It is favoured by large $h_{m}$, large $F_{d}$, and/or large $w$.

Significantly, our numerical simulations suggest that it is not possible to remove the left-hand wall from the problem and still be able to realize a hydraulically controlled flow. Even when the initial flow is separated and $w$ is large, the critical sill flow remains attached to the left-hand wall. In addition, upstream influence for large $w$ is transmitted in the form of an intrusion that travels along the left-hand wall. These results imply that a 'coastal' version of our current, set up by moving the left-hand wall to infinity, cannot be hydraulically controlled nor have a stationary hydraulic jump. One caveat should be mentioned: by restricting the initial conditions so as to require zero volume transport in the left-hand boundary $(\S 3.1)$ all separated initial flows are supercritical. There is another family of separated but subcritical initial flows that could conceivably be subject to upstream influence without the aid of the left-hand wall.

For some of the interesting features found in our simulations, no concrete oceanographic observations have been reported. Such features include the Kelvin-wave hydraulic jump (figure 16), the transverse hydraulic jump (figures 14 and 17), and the bifurcation of the flow approaching the sill with resulting leakage back into the upstream part of the channel (figure 15). However, it is notoriously difficult to make observation in deep channels such as the Denmark Strait and Faroe Bank Channel. These calculations should help investigators determine where to look and what to look for.

This work was initiated during the 1997 Geophysical Fluid Dynamics Summer Study Program 'Rotating Hydraulic Control' at the Woods Hole Oceanographic Institution. The authors have been supported by the National Science Foundation through Grants (OCE-9810599 for L.J.P. and K.R.H. and OCE-9711186 for EPC). L.J.P. also received support from the Office of Naval Research under Grant N0001495-1-0456 and K.R.H. under grant N00014-93-1-0263. The authors wish to thank Heather Deese and two anonymous reviewers for some helpful comments. This is Woods Hole Oceanographic Institution contribution 10034.

\section{REFERENCES}

BaInes, P. G. 1995 Topographic Effects in Stratified Flows. Cambridge University Press, 482 pp.

Baines, P. G. \& Davies, P. A. 1980 Laboratory studies of topographic effects in rotating and/or stratified fluids. In Orographic Effects in Planetary Flows. GARP Publication 23, WMO/ICSU, pp. 233-299.

BAines, P. G. \& LEONARD, B. P. 1989 The effects of rotation on flow of a single layer over a ridge. Q. J. R. Met. Soc. 115, 293-308 (referred to herein as BL).

BorenÄs, K. M. \& LundBerg, P. A. 1986 Rotating hydraulics of flow in a parabolic channel. J. Fluid Mech. 167, 309-326. 
Borenäs, K. M. \& Whitehead, J. A. 1998 Upstream separation in a rotating channel flow. J. Geophys. Res. 103, C4, 7567-7578.

Dalziel, S. B. 1990 Rotating two-layer sill flows. In The Physical Oceanography of Sea Straits (ed. L. Pratt), Kluwer, 587 pp.

Fedorov, A. V. \& Melville, W. K. 1996 Hydraulic jumps at boundaries in rotating fluids. J. Fluid Mech. 324, 55-82.

GiLl, A. E. 1977 The hydraulics of rotating-channel flow. J. Fluid Mech. 80, 641-671.

Gill, A. E. 1982 Atmospheric-Ocean Dynamics. Academic Press, 662 pp.

Haynes, P. H., Johnson, E. R. \& Hurst, R. G. 1993 A simple model of Rossby wave hydraulic behaviour. J. Fluid Mech. 253, 359-384.

Helfrich, K., Kuo, A. \& Pratt, L. J. 1999 Nonlinear Rossby adjustment in a channel. J. Fluid Mech. 390, 187-222.

Houghton, D. D. \& Kasahara, A. 1968 Non-linear shallow fluid flow over an isolated ridge. Commun. Pure Appl. Maths 21, 1-23.

Killworth, P. D. 1992 Flow properties in rotating, stratified hydraulics. J. Phys. Oceanogr. 22, 997-1017.

Killworth, P. D. 1994 On reduced-gravity flow through sills. Geophys. Astrophys. Fluid Dyn. 75, 91-106.

Killworth, P. D. 1995 Hydraulic control and maximal flow in rotating stratified hydraulics. Deep-Sea Res. 42, 859-872.

Killworth, P. D. \& McDonald, N. R. 1993 Maximal reduced-gravity flux in rotating hydraulics. Geophys. Astrophys. Fluid Dyn. 70, 31-40.

LeVeque, R. J. 1997 Wave propagation algorithms for multidimensional hyperbolic systems. J. Comput. Phys. 131, 327-353.

LoNG, R. R. 1954 Some aspects of the flow of stratified fluids. II Experiments with a two-fluid system. Tellus 6, 97-115.

LoNG, R. R. 1970 Blocking effects in flow over obstacles. Tellus 22, 471-480.

NoF, D. 1984 Shock waves in currents and outflows. J. Phys. Oceanogr. 14, 1683-1702.

NoF, D. 1986 Geostrophic shock waves. J. Phys. Oceanogr. 16, 886-901.

OrLANSKI, I. 1976 A simple boundary condition for unbounded hyperbolic flows. J. Comput. Phys. 21, 251-269.

Paldor, N. 1983 Stability and stable modes of coastal fronts. Geophys. Astrophys. Fluid Dyn. 27, 217-228.

Pratt, L. J. 1983 On inertial flow over topography. Part 1. Semigeostrophic adjustment to an obstacle. J. Fluid Mech. 145, 195-218.

PratT, L. J. 1987 Rotating shocks in a separated laboratory channel flow. J. Phys. Oceanogr. 17, 483-491.

Pratt, L. J. \& ARMi, L. 1987 Hydraulic control of flows with nonuniform potential vorticity. J. Phys. Oceanogr. 17, 2016-2029.

Pratt, L. J. \& Armi, L. 1990 Two-layer rotating hydraulics: strangulation, remote and virtual controls. Pure Appl. Geophys. 133, 587-617.

Pratt, L. J. \& Chechelnitsky M. 1997 Principles for capturing the upstream effects of deep sills in low resolution ocean models. Dyn. Atmos. Oceans 26, 1-25.

Pratt, L. J. \& Lundberg, P. A. 1991 Hydraulics of strait and sill flow. Ann. Rev. Fluid Mech. 23, 81-106.

Schär, C. \& Sмiтh, R. B. 1993 Shallow-water flow past isolated topography. Part I: Vorticity production and wake formation. J. Atmos. Sci. 50, 1373-1400.

SHEN, C. 1981 The rotating hydraulics of open-channel flow between two basins. J. Fluid Mech. 112, $161-188$.

Smolarkiewicz, P. K. \& Clark, T. L. 1986 The multidimensional positive definite advection transport algorithm: Further development and applications. J. Comput. Phys. 67, 396-438.

Stern, M. E. 1974 Comment on rotating hydraulics. Geophys. Fluid Dyn. 6, 127-130.

StERn, M. E. 1980 Geostrophic fronts, bores, breaking and blocking waves. J. Fluid Mech. 99, 687-703.

Whitehead, J. A., LeetmaA, A. \& Knox, R. A. 1974 Rotating hydraulics of strait and sill flows. Geophys. Fluid Dyn. 6, 101-125. 